\title{
Aptamer Functionalized Zinc Oxide Field Effect Transistors For Odor Detection
}

\author{
Michael D. Aldridge \\ West Virginia University, maldrid2@mix.wvu.edu
}

Follow this and additional works at: https://researchrepository.wvu.edu/etd

Part of the Electronic Devices and Semiconductor Manufacturing Commons, Other Biomedical Engineering and Bioengineering Commons, and the Other Neuroscience and Neurobiology Commons

\section{Recommended Citation}

Aldridge, Michael D., "Aptamer Functionalized Zinc Oxide Field Effect Transistors For Odor Detection" (2019). Graduate Theses, Dissertations, and Problem Reports. 3932.

https://researchrepository.wvu.edu/etd/3932

This Thesis is protected by copyright and/or related rights. It has been brought to you by the The Research Repository @ WVU with permission from the rights-holder(s). You are free to use this Thesis in any way that is permitted by the copyright and related rights legislation that applies to your use. For other uses you must obtain permission from the rights-holder(s) directly, unless additional rights are indicated by a Creative Commons license in the record and/ or on the work itself. This Thesis has been accepted for inclusion in WVU Graduate Theses, Dissertations, and Problem Reports collection by an authorized administrator of The Research Repository @ WVU. For more information, please contact researchrepository@mail.wvu.edu. 


\title{
APTAMER FUNCTIONALIZED ZINC OXIDE FIELD EFFECT TRANSISTORS FOR ODOR DETECTION
}

\author{
Michael Aldridge \\ Thesis submitted to the Eberly College of Arts and Sciences \\ at West Virginia University \\ in partial fulfillment of the requirements \\ for the degree of \\ Master of Science \\ in \\ Biology
}

Kevin C. Daly, Ph.D., Committee Chairperson

Andrew Dacks, Ph.D.

Biology

Cerasela Zoica Dinu Ph.D.

Chemical and Biomedical Engineering

Morgantown, West Virginia

2019

Keywords: Electronic Nose, Aptamer, Zinc Oxide, Field Effect Transistor, Chemical Sensing, Olfaction

Copyright 2019 Michael Aldridge 


\begin{abstract}
APTAMER FUNCTIONALIZED ZINC OXIDE FIELD EFFECT TRANSISTORS FOR ODOR DETECTION IN AIR
\end{abstract}

\begin{abstract}
Michael Aldridge
Odor detection and identification are complex processes, and tasks that currently only animals do well. There is a pressing need for an electronic nose, or eNose, with good sensitivity, selectivity, and speed that mimics that ability. Food quality control operations, environmental sensing, occupational safety, and the defense sectors all require systems that can rapidly and reliably detect trace levels of volatile organic compounds. Although recent development in gas-phase chemical sensors has enabled the detection of trace quantities of such chemicals, there is currently no device which can detect the broad range of odorants as effectively as most animals. This is partly due to a gap between what we know about naturally occurring olfactory systems and the implementation of that knowledge for the design of artificial olfactory systems. The goal of this work is to create a biologically inspired device which can accurately detect and identify odors at concentrations consistent with the most sensitive biological systems.

In order to mimic a natural olfactory system, we replaced the biological components of the olfactory system with synthetic components. For example, the device is contained on a silicon chip instead of within olfactory epithelium. Individual olfactory receptor proteins are replaced by aptamers, which are short sequences of single-stranded DNA, identified via an evolution-based process, to bind a particular target. Like olfactory receptor proteins, aptamers can bind more than one homologous molecule. If successful, this approach would provide a basis for a computational strategy that identifies odor based on combinatorial patterns of receptor activation, so that the number of recognizable odors exceeds the number of receptors.

Our efforts produced an aptamer-decorated zinc oxide field effect transistor (Apta-FET) that shows great sensitivity to target compounds, but limited selectivity. We demonstrated that aptamers attached to a FET respond to targets in a concentrationdependent manner, and that this response can be measured electrically. The limited selectivity of our device highlights the need for many different kinds of aptamers within the same device, as well as the need for more advanced computational analysis of the output data. It is our hope that these hurdles will be overcome.
\end{abstract}




\section{DEDICATION}

The author wishes to thank Allen and Janis Aldridge for their loving help and support during the research and graduate school process.

The author would also like to thank Alycia Knepp, Darren Power, Zachary Rowe, Cody Sickels, Nathan Kinsley, Eric Kinsley, Philip Kirk, Barbara White, Candice Cooley, Justin Cooley, Llew Williams, Rick Stadtmiller, Michael Thobois, Russell Wyatt, Patrick Johns, Dano McCawley, and many other close friends and family who helped on a daily basis while completing this degree. 


\section{ACKNOWLEDGMENTS}

The author wishes to acknowledge use of the WVU Shared Research Facilities in facilitating completion of this work. Harley Hart was an invaluable resource, and this work would not have been possible without his assistance guidance. The author wishes to acknowledge the support of the NanoSAFE (formerly WVU Nano) fellowship program. The author also wishes to acknowledge Letha Sooter, Jessica Lear, and the Sooter Lab for performing aptamer selections and providing the sequence data for the cadaverine aptamer and the methyl ethyl ketone aptamer. In addition, the author acknowledges David Lederman, the Lederman Lab, and the WVU Department of Physics, who provided exceptional support and accommodation for the duration of this work. 


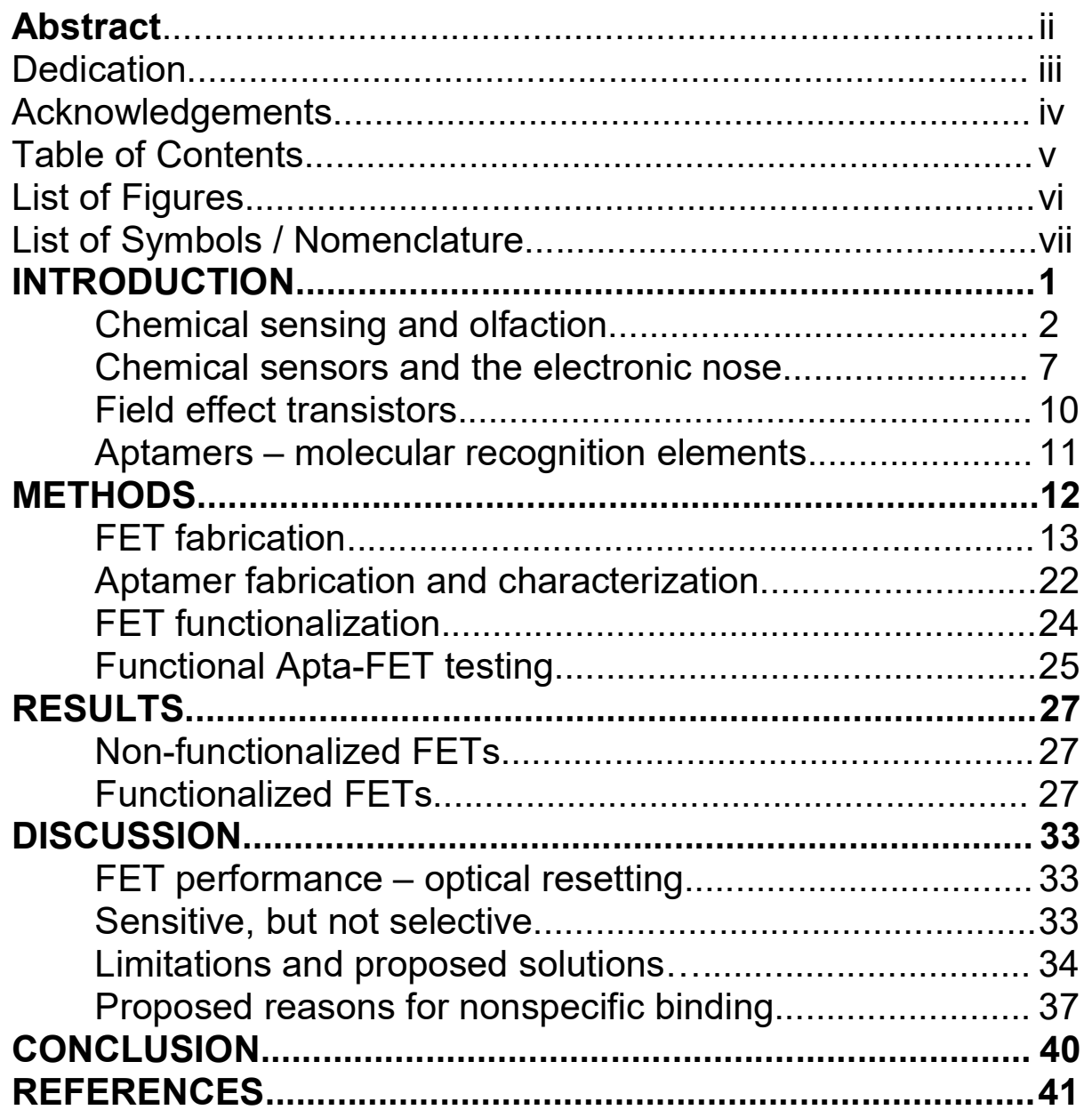




\section{LIST OF FIGURES}

Figure 1. ZnO FET processing and production ..............................................15

Figure 2. FET pattern using shadow masks ..................................................18

Figure 3. Photolithography and E-beam Lithography .......................................19

Figure 4. Output $(A)$ and transfer $(B)$ measurements .................................28

Figure 5. Preliminary electrical measurements of an Apta-FET ..........................29

Figure 6. Functional testing procedure .................................................... 30

Figure 7. Functional testing paradigm for multi-analyte testing ..........................31

Figure 8. Response of Apta-FETs ZnO20160503A and ZnO20160503B .............31

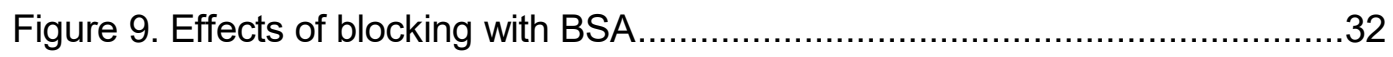




\begin{tabular}{|c|c|}
\hline AMP & Adenosine monophosphate \\
\hline ATP & Adenosine triphosphate \\
\hline BOE & Buffered oxide etch \\
\hline BSA & Bovine serum albumin \\
\hline $\mathrm{DI}$ & Deionized (DI $\mathrm{H}_{2} \mathrm{O}$, deionized water) \\
\hline FET & Field effect transistor \\
\hline GPCR & G-protein coupled receptor \\
\hline $\mathrm{HF}$ & Hydrofluoric acid \\
\hline IR & Ionotropic receptor \\
\hline MEK & Methyl ethyl ketone (2-butanone) \\
\hline $\mathrm{mT}$ & miliTorr $\left(10^{-4}\right.$ Torr $)$ unit of pressure \\
\hline$\mu \mathrm{m}$ & Micrometers, $1 \mu \mathrm{m}=1 \times 10^{-6}$ meters \\
\hline$\mu \mathrm{M}$ & Micromolar, $1 \mu \mathrm{m}=1 \times 10^{-6} \mathrm{mols} / \mathrm{L}$ \\
\hline n-doped & Negatively doped (anion doped) \\
\hline $\mathrm{nm}$ & Nanometers, $1 \mathrm{~nm}=1 \times 10^{-9}$ meters \\
\hline $\mathrm{nM}$ & Nanomolar, $1 \mathrm{~nm}=1 \times 10^{-9}$ mols $/ \mathrm{L}$ \\
\hline OBP & Odorant binding protein \\
\hline ORN & Olfactory receptor neuron \\
\hline OR & Olfactory receptor protein \\
\hline p-doped & Positively doped (cation doped) \\
\hline PCR & Polymerase chain reaction \\
\hline RF & Radio frequency \\
\hline SAW & Surface acoustic wave \\
\hline SELEX & Selective evolution of ligands by exponential enrichment \\
\hline V & Volts \\
\hline $\mathrm{V}_{\mathrm{DS}}$ & Drain-Source voltage \\
\hline$V_{G}$ & Gate voltage \\
\hline XRD & X-ray diffraction \\
\hline XRR & X-ray reflectivity \\
\hline$\Omega$ & Ohms, a measure of electrical resistance \\
\hline$\Omega \cdot \mathrm{cm}$ & Ohm-centimeters, a measure of resistivity \\
\hline
\end{tabular}




\section{Introduction}

The design and production of chemical sensors and electronic nose devices have, in recent years, made great advances (Röck et al., 2008; Wilson and Baietto 2009). However, these devices are still inferior to the olfactory systems of many animals because they lack receptor diversity, sufficient response specificity, and speed (Wilson and Baietto 2009). To this end, we aimed to design and fabricate a single receptor channel detector device that was fast, sensitive and selective, using principles found in nature. Specifically, we fabricated a device which would act as a single olfactory receptor.

The device produced relied on the binding of two molecules, an aptamer and a target. In order to detect this binding event, a suitable platform or transducer was needed. To this end, a thinfilm field effect transistor (FET) was fabricated, and aptamers attached to the active surface channel of the device. It was proposed that the structural change in the aptamer after binding would induce a change in the electrical field of the FET, and thus change the amount of current flowing through the device.

The product of this work was two aptamer-decorated field effect transistors (Apta-FETs), one with sensitivity to cadaverine, and the other to methyl ethyl ketone (MEK). These small amines were chosen because of their relevance in health and safety. Cadaverine is an unpleasant odorant that is produced by the decomposition of animal tissue. Detection of cadaverine is therefore important for military and law enforcement efforts in locating the deceased. MEK, in addition to being a biologically relevant compound in the detection of certain cancers, is a hazardous chemical encountered in occupational settings (NIOSH 1988). We were successful in developing a device with high sensitivity to the target analytes in vitro, but with limited discriminatory power. 


\section{A. Chemical sensing and olfaction}

Chemical sensing is one of the most basic ways that an organism receives information about its surroundings. Evolutionarily speaking, it is likely the oldest form of sensation, and nearly every organism possesses some method of chemical detection (Brennan and Keverne 2004). This detection is achieved when a volatile molecule, or odorant, is perceived by the organism in some meaningful way.

The chemical environment around an organism contains a wealth of information (Bear et al., 2007). The ability to find food sources (Distel and Hudson 1985), avoid predation (Dorries et al., 1997), or locate and select a mate (Novotny 2003) typically relies on olfactory cues. For most single celled organisms, chemical sensing is the only method by which any of these abilities are possible.

Individual cells within a multicellular organism use chemical sensing and signaling for many purposes as well: axonal growth cones in neurons use chemical cues during development (Mortimer et al., 2008), cells in the heart respond to epinephrine (Gordan et al., 2015), and osteoblasts and osteoclasts respond to hormones to either deposit or digest bone (Chen et al., 2018). There are countless examples of chemical signaling between cells within a multicellular organism, but our focus is on olfaction - the event by which an animal detects and identifies volatile chemical compounds (odors) in its external environment.

In animals, olfaction at the organismal level relies on the binding of odor molecules to special proteins called olfactory receptor proteins (ORs; Mombaerts 2004). These proteins are expressed by olfactory receptor neurons (ORNs), which both act as the molecular recognition 
element and the transducer that converts the chemical binding event into an electrochemical signal.

Olfactory receptor proteins are typically 7-transmembrane spanning, G-protein coupled receptors that are embedded in the membrane of ORNs (Bear et al., 2007; Peter 2004; Hoon et al., 1999). The canonical ORs are 7-transmembrane G-protein coupled receptors (GPCRs), and are responsible for the transduction of a chemical odor signal to an electrochemical signal in an ORN.Buck and Axel (1991), discovered that ORs were encoded by a distinct super family of genes in rodents. Their work demonstrated that this multigene family had certain characteristics which suggested that these proteins were responsible for odor transduction. First, each encoded protein had a variable extracellular region, which fits with the model of structurally diverse odorant molecules binding to proteins on the outside of a cell. Second, these proteins have seven-transmembrane spanning regions, which indicates that they are membrane bound proteins. Third, the cytoplasmic side of the proteins are more conserved, suggesting that they are all capable of interacting with an intercellular cascade mechanism to complete the transduction process. In our work, we sought to mimic the ligand specificity of the extracellular domain of these ORs using DNA aptamers, which varied in sequence. Attaching these aptamers to the same zinc oxide FET parallels the intercellular signaling cascade, with the goal of creating a measurable electrical signal in the form of an effective change in the gate field.

Vosshall et al., (1999) further explored this gene family in Drosophila, and showed that expression of these proteins was largely confined to olfactory receptor neurons in the antenna. Furthermore, by mapping expression of these proteins in the antenna, they demonstrated that each neuron expresses different combinations of ORs. In 2004, Larsson and colleagues identified that a particular Drosophila odorant receptor, OR83b, which is expressed ubiquitously 
in ORNs. They showed that deletion of this OR resulted in the failure of the olfactory system. This indicates that the OR83b, the so called "ORCO" is a required coreceptor.

Over a decade later, a different family of olfactory receptor proteins was described (Benton et al., 2009). In contrast to the metabotropic GPCRs described earlier, these proteins are ionotropic, and directly change the conductance of the ORN membrane as a result of odorant binding. Like metabotropic ORs, these IRs exhibit high variability in the ligand-binding region. However, their structure around the ion channel is similar to ionotropic glutamate receptors, which indicates that they retain their ability to conduct ions. This is similar to the genetic divergence and conservation in other olfactory receptor proteins, in that the extracellular domain exhibits a high variability, presumably to interact with the diversity of odorant molecules, while the cytoplasmic or membrane regions are conserved, enabling the protein to effectively interact with cellular machinery. Thus, the ability of these membrane bound receptors to effectively transduce the odorant binding event into a meaningful cellular signal is rooted in their genetic makeup. Therefore, it is critical that a variety of different aptamer sequences be incorporated to reflect the sequence diversity of the OR extracellular domain.

In addition to ionotropic receptors (IRs) and GPCRs, there are odorant binding proteins (OBPs) that are associated with the support cells surrounding ORs (Larter et al., 2016). While previously suspected to enable or support odorant transport to the olfactory receptor proteins, Larter and colleagues showed that they may act as a buffer, or mediate the interaction of odorants and ORs in another manner. While we did not incorporate a secondary binding molecule, adding a component to this effect could enhance the performance of our device in future work.

When an odorant molecule binds to an olfactory receptor protein, it causes a membrane depolarization, and potentially an action potential in the olfactory receptor neuron. For 
mammalian metabotropic ORs, the binding of an odorant to the extracellular domain of the receptor results in the recruitment of adenylyl cyclase to the receptor, which converts adenosine triphosophate (ATP) into cyclic adenosine monophosphate (cAMP). The rise in intracellular cAMP opens a cyclic gated sodium channel, which results in an influx of $\mathrm{Na}+$ and potentially an action potential (Buck and Axel 1991). Insect ORs are distinct in that they contain both ionotropic and metabotropic response components (Wicher et al., 2008). For ionotropic component of the OR response, the binding of an odorant results in the opening of a central pore in the protein, increasing the permeability of the membrane to cations and causing an action potential through depolarization (Rytz et al., 2013, Wicher et al., 2008).

This transduction mechanism, from odorant molecule to action potential, and even odor perception, was extensively investigated by Hallem and Carlson (2006) by exposing Drosophila to a battery of over 100 odors. What they found is that while some ORs respond to a single or small number of odor molecules, others respond to many different odor molecules. These narrowly tuned, or highly selective receptors, may respond to only one odorant. The broadly tuned receptors, on the other hand, tend to be sensitive to a particular class of structurally similar molecules, such as alcohols, esters, or amines (Hallem and Carlson 2006). A similar profile is reported for inhibitory responses. In fact, inhibitory responses from odorants appear to occur more frequently than excitatory responses in the receptors tested (Hallem and Carlson 2006). Furthermore, the different ORs tested show tuning curves that are broadly tuned, narrowly tuned, or anywhere in between. At lower concentrations, the more broadly tuned receptors responded to fewer odorants indicating that tuning is concentration dependent (Hallem and Carlson 2006).

Therefore, in natural olfactory systems, there is a continuous distribution from narrowly tuned, highly specific, receptors, to broadly tuned, less specific, receptors. This greatly increases the 
number of analytes which can be detected with a limited number of sensors, because each chemical stimulus results in the activation of different combinations of receptors (Hallem and Carlson 2006). The aptamers presented in this work represent broadly tuned ORs, in that they ultimately were highly sensitive but with limited selectivity. These broadly tuned aptamers are an important part of a robust olfactory response, and should be incorporated into future devices. Moreover, the SELEX process is amenable to producing aptamers with different tuning properties, which holds the promise for the generation of an aptamer library with different degrees of affinity/specificity to a variety of compounds (Ellington and Szostak 1992; Klug and Famulok 1994; Knight and Yarus 2003; Tuerk and Gold 1990; Joyce 1989).

Despite the ability to detect odorants in air, a gaseous medium, natural olfactory systems require a liquid interface in order to function. (Steinbrecht 1998; Pelosi 1995; Menco and Farbman 1992) This mucosa functions to protect the olfactory receptors from desiccation, and also contains numerous odorant binding proteins (OBPs) (Later et al., 2016; Briand et al., 2000; Park et al., 2000; Dear et al., 1991). The role of these OBPs is not just simply to transport hydropobic odorants across the aqueous mucosa (Pelosi 1995; Steinbrecht 1998, Larter et al., 2016). These OBPs, along with detoxifying enzymes in the mucosa, have been proposed to deactivate the odorant response (Pelosi 1995). They have also been proposed to bind the odorant in such a way as to make it more recognizable to the olfactory receptor protein (Prestwich 1996). More recently, OBPs in Drosophila have been shown to be important for normal olfactory function (Larter et al., 2016). However, Larter and colleagues found a robust response from an olfactory sensillum that was devoid of any OBP. They demonstrated that OBPs serve to enhance the selectivity for a target odorant in the presence of a background. These proteins thus serve to filter and buffer the local mucous environment around olfactory sensilla and play a more nuanced role than previously thought. 
The Apta-FET we fabricated was exposed to chemical in an aqueous solution to approximate this aspect of the ORN environment but was dried prior to testing. Incorporating a mucous or wet polymer layer may be possible in future devices, but was not considered within the scope of the current work. However, it may be a critical part of a dynamic and flexible olfactory system.

\section{$\underline{\text { I. B. Chemical sensors and the electronic nose }}$}

In general, sensory systems facilitate a flow of information from the external environment to a central processing and decision-making entity. In biological systems, this is the brain, or more specifically, higher brain regions. In man-made systems, this central decision-maker can be an output monitor that informs the user, or software that is programmed to respond to a stimulus. In both cases, the stimulus must be detected, converted or transduced into a meaningful signal, and then processed. For example, the flow of information in a word processing program - from the typist's fingers to the words on the screen - follows the same general outline. The detector (key) receives a physical force which is then transduced into an electrical signal by electronics in the keyboard. The signal (“type the letter 'm") is processed by the software. In a biological olfactory system, the ORs detect the presence of volatile compounds (detector). This detection event is transduced into an electrical signal by a cascade of protein interactions around the OR, along with the ORN itself (transducer). This signal is carried by the ORN where it is processed at the glomerular level and passed to higher brain regions (processor).

An electronic nose works using the same principles of detection, transduction, and processing (Persaud and Dodd 1982; Röck et al., 2008; Mitrovics et al., 1998). There are a wide variety of detectors (Wilson and Baietto 2009); the primary function of these detectors (the keys of the keyboard) is to respond to the presence of a gas or volatile compound that they are sensitive to. These are attached to or incorporated into a transducer element which converts the detection 
response to an electrical signal - typically a change in resistance (Nylander 1983; Bai et al., 2007; Yamada et al., 2001), capacitance (Plum et al., 2006; Braik et al., 2015; Hagleitner 2002), voltage (Lin and Tseng 1998), or current (Wang et al., 1998). The metal oxide semiconductor field effect transistor (MOS-FET, hereafter simply FET) is a popular transduction element (Yamazoe et al., 1983; Winquist et al., 1985; Åbom et al., 2002). One of the primary challenges in electronic nose technology is the processing of these electrical signals (Gao et al., 2012). While we designed an electronic nose "receptor" that mimics the recognition and transduction processes of an olfactory receptor protein and ORN, addressing the processing problem was beyond the scope of the current thesis.

Detector elements in electronic chemical sensing systems rely on chemical interactions between a test gas and a sensor element. Typically, these chemical interactions modulate the way that the sensor element behaves in an electronic circuit. Changes in resistance and capacitance are commonly exploited (Nylander 1983; Bai et al., 2007; Yamada et al., 2001; Plum et al., 2006; Braik et al., 2015; Hagleitner 2002). For many detector elements, the detection and transduction step are combined. There are examples of detector elements which separate these steps - surface acoustic wave (SAW) sensors (Drafts 2001), for example, detect the mass of elements absorbed on the surface of the sensor (Abdollahi et al., 2007). By coating a SAW device with a selective element, such a polymer, it is possible to selectively capture a target analyte (Wohltjen and Dessy 1979; Alizadeh and Zeynali 2008). Since SAW devices are able to detect minute changes in mass, the amount of added mass can be used to determine the concentration of analyte adsorbed. This principle is also employed by quartz microbalance sensors (Lucklum et al., 1991; Grate 2000) and cantilever mass balance sensors (Battiston et al., 2001). 
The first semiconductor gas sensor, invented by Naoyoshi Taguchi in 1972, took advantage of a resistivity change in a semiconductor upon exposure to a gas (U.S. Patent No. 3,695,848, 1972). In 1975, Lundström et al., reported metal-oxide semiconductor FETs which responded to hydrogen. Building on this, metal oxide sensors operate on the principle that certain gas species react with certain metal oxides in a reproducible way (Persaud and Dodd 1982). These sensors often rely on a chemical reaction between the gas to be detected and the metal oxide. Typically, the metal oxides chosen reflect the target analyte, and there is some degree of $a b$ initio selection of target gasses. The array of sensors is then exposed to a test library of vapors and gasses, from which a response library of "fingerprints" can be obtained as a combinatorial activation pattern (Gao et al., 2012). The drawbacks to this technology are that these sensors are biased strongly by humidity and oxygen content (Wilson and Baietto 2009).

Sensor arrays which incorporate conductive polymers operate on a similar principle as metal oxide sensor arrays (Wilson and Baietto 2009; Plum et al., 2006; Braik et al., 2015). Variations in chemistry and physical properties of certain polymers result in different responses to different vapors and gasses. In addition, polymer composition and thickness can affect diffusion of the analyte into the sensor material, adding another layer of complexity which can aid in the differentiation of test samples. Like metal oxide sensor arrays, the selectivity of a conductive polymer array is dependent on the size and complexity of the training library. In addition, temperature and humidity can create large biases in the response signals. Modern electronic nose devices typically incorporate both metal oxides and conductive polymer sensors (Wilson and Baietto 2009). 


\section{$\underline{\text { I. C. Field effect transistors }}$}

The field effect transistor is an electronic device in which the flow of current between two terminals, the source (S) and drain (D) is enhanced or diminished by the application of voltage across a third terminal, the gate (G) (U.S. Patent No. 3,102,230, 1963; Bayraktaroglu et al., 2008).

The application of a voltage to the gate terminal (the gate voltage, $V_{G}$ ) creates an electrical field, the eponymous field effect, in the material between the source and the drain. For a set voltage drop across the source and drain (the bias voltage, $V_{D S}$ ) a change in $V_{G}$ produces a change in the output current (U.S. Patent No. 3,102,230, 1963).

FETs are good transducers for sensor devices because a small change in gate voltage, or the field, can produce large changes in the source-drain current (Winquist et al., 1985; U.S. Patent No. $3,102,230,1963)$. Depending on the properties of the FET, a change in gate voltage facilitates or impedes electrical current flow from the source to the drain. In this manner, FETs can act as signal amplifiers (Carcia et al., 2003). In designing and assembling our device, the field effect transistor was used to convert the binding event of the molecular recognition element of the detector to the analyte into a measurable electrical signal. Here, we developed and used aptamers (described below) as the molecular recognition element.

Transistor performance is based on two types of measurements, transfer and output. In both of these measurement modes, the current flowing between the source and the drain is measured (IDS). For transfer measurements, the bias voltage is held constant while different gate voltages

are applied. Typically, the lowest expected gate voltage is applied first, current is measured, and the gate voltage is increased in a stepwise manner until the highest expected gate voltage is 
reached. This produces a series of bias currents relative to gate voltage. Typically, this process is repeated at several bias voltages, and a set of transfer curves is obtained (Carcia et al., 2003; Bayraktaroglu et al., 2008).

Output measurements, in contrast, hold the gate voltage constant while different bias voltages are applied. Each output measurement produces a series of bias currents relative to bias voltages at a single gate voltage. Again, this process is typically repeated for a set of gate voltages (Carcia et al., 2003; Bayraktaroglu et al., 2008).

\section{$\underline{\text { I. D. Aptamers - molecular recognition elements }}$}

Aptamers are short (20-50 base pairs) nucleic acid oligomers which bind strongly to a target of interest (Ellington and Szostak 1992; Klug and Famulok 1994; Knight and Yarus 2003; Tuerk and Gold 1990; Joyce 1989). In our work, we identified and used single stranded DNA aptamers. The binding properties of an aptamer depend directly on the DNA sequence, which also determines its shape and structure in space. The sequence of the single-stranded DNA aptamers determines their shape because different base pairs interact with each other, in part by base pair hybridization. The sequence-determined shape of an aptamer allows it to bind to target analytes using van der waals and other small intermolecular forces (Klug and Famulok 1994; Feigon et al., 1996). This binding can be very strong $(\mathrm{Kd}<10 \mu \mathrm{M})($ Sassanfar and Szostak 1993) for some aptamer-target complexes. This is similar to antibody-antigen interactions $(\mathrm{Kd}=200 \mu \mathrm{M})$ (Moutin et al., 1994). In our case, the appropriate sequence for a target-specific aptamer is identified using the SELEX process (Selective Evolution of Ligands by EXponential enrichment), an in-vitro selection method (Klug and Famulok 1994). 
Aptamers were selected using the SELEX, an iterative process in which a library of single stranded DNA (ssDNA), or in some cases, double-stranded DNA or RNA sequences of a specific length are incubated with a target molecule, selected for their ability to bind to that molecule, and amplified (Klug and Famulok 1994; Knight and Yarus 2003; Tuerk and Gold 1990; Joyce 1989). Since the aptamers themselves are short pieces of ssDNA, they can be amplified using the polymerase chain reaction (PCR). After numerous rounds of incubation, selection, and amplification, the pool becomes enriched with oligonucleotides that have good binding affinity for the target molecule. The remaining pool is sequenced, and aptamers are identified which have good binding affinity for a selected target, or other desirable properties.

The present study takes advantage of aptamers identified by collaborators in the laboratory of Dr. Letha Sooter. Their work in selecting aptamers for cadaverine and methyl ethyl ketone (MEK) was pioneering because the targets were small molecules not readily immobilized on a substrate. To overcome this, they used capillary electrophoresis (CE) SELEX (Mosing and Bowser 2009). Capillary electrophoresis separates molecules or constructs in solution based on their charge and hydrodynamic radius, eliminating the need to bind the aptamers or target molecules to a substrate.

\section{Methods}

In the present work, ssDNA aptamers we used were selected for affinity to cadaverine and methyl ethyl ketone (MEK or 2-butanone), which are both small, biogenic, amines. In addition, we purchased commercially available aptmers with high affinity for ATP (Huizenga and Szostak 1995) to use as a positive control. The finished device consisted of a field effect transistor decorated with aptamers. These devices were fabricated using RF magnetron sputtering (Carcia et al., 2003) for a Zinc Oxide (ZnO) semiconductor layer and DC sputtering to deposit 
metallic contacts. Shadow masks were used to pattern the $\mathrm{ZnO}$ and metals. Aptamers were attached to the $\mathrm{ZnO}$ surface using silane chemistry (Hagen et al., 2010).

\section{A. FET fabrication}

FETs were fabricated on Si/SiO2 substrates. Blank Si (Silicon Quest International) was p-doped (Boron), $<100>$, with a thickness of $525 \pm 25 \mu \mathrm{m}$. "<100>" refers to the orientation of the crystallographic plane in the wafer. Dry thermal oxidation was performed by the manufacturer to create a $\mathrm{SiO}_{2}$ layer on both sides of the wafer with a thickness of $2000 \AA \pm 5 \%$. The wafers were single-side polished, and heavily doped $(0.005 \Omega-\mathrm{cm})$. The high conductivity of the substrates was critical, since the bulk silicon acted as the back gate electrode for the device.

Wafers were prepared by etching the oxide layer from the unpolished surface using buffered oxide etch (BOE, Microchem Inc.), an HF-containing solution for dissolving $\mathrm{SiO}_{2}$. This was necessary in order to expose the bare silicon surface, which is conductive. Removing the insulating $\mathrm{SiO}_{2}$ layer allowed us to make electrical contact with the bulk silicon. The polished surface was protected by a layer of AZ-5214 photoresist (Microchem Inc.) applied using spincoating. Removal of the oxide layer was confirmed visually based on hydrophobicity and conductivity was confirmed using a multimeter. This was possible because $\mathrm{SiO}_{2}$ is hydrophilic, but $\mathrm{Si}$ is hydrophobic. Thus, the absence of $\mathrm{SiO} 2$ was easy to observe by spraying water on the wafer and observing the degree of wetting.

To protect the etched surface from oxidation, wafers were immediately coated with $\mathrm{Ti} / \mathrm{Au}$ using DC sputtering (CVC 610 DC Magnetron Sputtering Station) or electron beam evaporation (Temescal BJD 2000 E-Beam Evaporator). Finally, wafers were diced into 10mm x 10mm chips using a DISCOTECH DAD-3240 Dicing Saw. 
$\mathrm{ZnO}$ was deposited using RF (radio frequency) magnetron sputtering (Carcia et al., 2003), and patterned using a shadow mask (Fig. 1A-C). Sputtering is the use of an electrical bias between the sample and the target to cause ions in the target material $(\mathrm{ZnO})$ to discharge and form plasma. RF sputtering employs an alternating current, which is necessary for insulating materials such as zinc oxide. Our system also uses a magnetron, which adds a magnetic field that enhances the deposition.

To determine the optimal growth conditions, a series of $\mathrm{ZnO}$ films were grown on $\mathrm{SiO}_{2}$ substrates and examined using $x$-ray reflectivity (XRR) for thickness (Fig. 1E) and x-ray diffraction (XRD) to determine crystallinity. Atomic force microscopy (AFM) was used to confirm roughness (Fig. 1D). Room-temperature films were grown at 15, 30, and 60 minutes to obtain an initial growth rate. A series of films were then grown at different substrate temperatures, using $23^{\circ} \mathrm{C}$ (room temperature), $100^{\circ} \mathrm{C}, 150^{\circ} \mathrm{C}, 200^{\circ} \mathrm{C}, 250^{\circ} \mathrm{C}$, and $300^{\circ} \mathrm{C}$ (Fig. 1F). Using XRD, we determined that the $300^{\circ} \mathrm{C}$ films exhibited the wurtzite crystal structure, which is desirable for a transistor (Bayraktaroglu and Leedy 2008).

For the final devices, 120W RF power was applied to a pure, 2" ZnO target. Substrates were heated to $300^{\circ} \mathrm{C}$ in vacuum and allowed to anneal for $1 \mathrm{~h}$ prior to deposition. The deposition was carried out in $\mathrm{O}_{2}$ and $\mathrm{Ar}$, at a pressure of $14 \mathrm{mT}$. A stoichiometric ratio of $66.6 \% \mathrm{O}_{2}$ : Ar was used, resulting in flow rates of $5.0 \mathrm{sccm}$ for $\mathrm{Ar}$ and $2.1 \mathrm{sccm} \mathrm{O}$. To achieve the desired thickness of $70 \mathrm{~nm}$, the deposition was carried out for 7200 s. During the deposition, the substrates were rotated at 23 RPM. The films were allowed to cool in vacuum for several hours prior to removal. After $\mathrm{ZnO}$ deposition, the devices were prepared with a second set of shadow masks to pattern electrical contacts (Fig. 1A). 

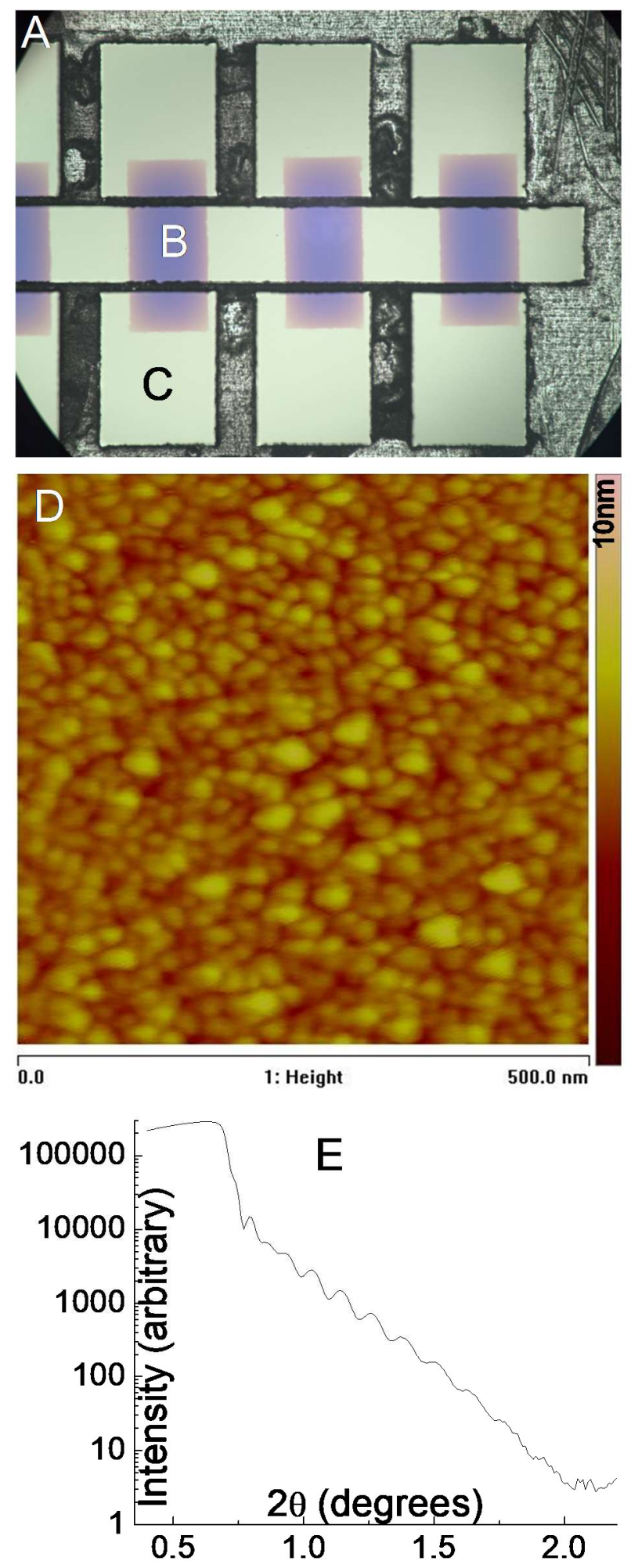

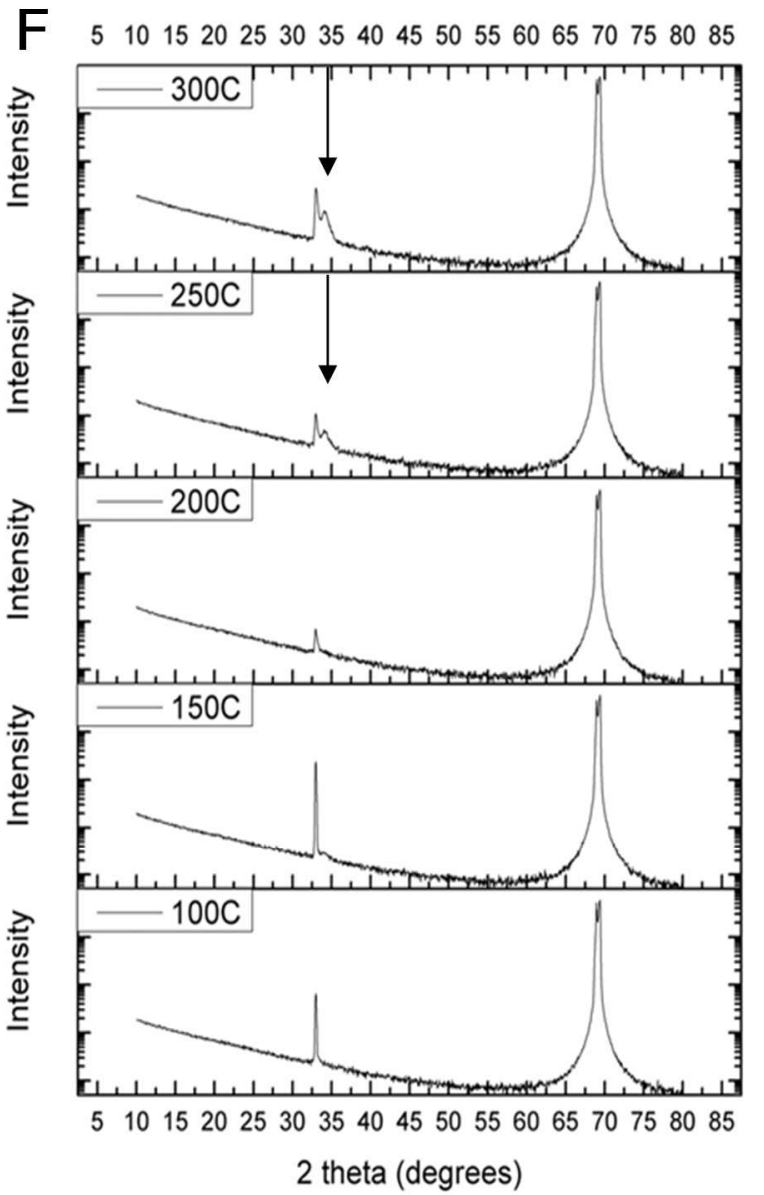

Figure 1. ZnO FET processing and production.

(A) The shadow mask for electrical contacts is placed over (B) the previously patterned $\mathrm{ZnO}$. (C) Lighter areas shown are the open areas of the shadow mask, which will allow $\mathrm{Al}$ and $\mathrm{Pt}$ to be deposited by DC sputtering. (D) Representative AFM image from a sputtered $\mathrm{ZnO}$ thin film. Using AFM, film roughness and surface geometry can be determined. The sample shown was sputtered at $200 \mathrm{C}$, and had a surface roughness of 0.512 $\mathrm{nm}$. (E) Representative low-angle X-ray reflectivity measurement for a sputtered ZnO film. Thickness is calculated using the $2 \theta$ position of the minima. The sample shown is $60 \mathrm{~nm}$ thick. (F) XRD measurements for ZnO films. Black arrows highlight the peak near $34^{\circ}$ which is indicative of wurtzite $\mathrm{ZnO}$. Sharp peaks near $33^{\circ}$ and $68^{\circ}$ are from the Si substrate. 
Initially, Ti/Au contacts were used to create an electrical junction with the $\mathrm{ZnO}$ thin films. However, these contacts proved unusable because the $\mathrm{Ti} / \mathrm{ZnO}$ interface was found to be insulating. We attempted to ameliorate this by using $\mathrm{Al} / \mathrm{ZnO}$ contacts, and contacts were made using $\mathrm{ZnO} / \mathrm{Al} / \mathrm{Au}$. Again, these contacts failed, because of a little-investigated and neglected issue known as "purple plague" which forms at Al/Au interfaces, creating an insulator. (Footner et al., 1987). Finally, we achieved low resistance electrical contact with $\mathrm{ZnO} / \mathrm{Al} / \mathrm{Ti} / \mathrm{Au}$ contacts then later switched to $\mathrm{ZnO} / \mathrm{Al} / \mathrm{Pt}$ contacts, which also resulted in a low resistance contact, but was a more practical approach.

Metals were deposited in the same sputter chamber, using pure Al and Pt targets. DC sputtering was performed at $23^{\circ} \mathrm{C}$ in Argon (5.0 sccm, 3.0mTorr). Al was sputtered for 300 s for a thickness of $\sim 50 \AA$, and Pt was sputtered for 120 s for a thickness of $\sim 950 \AA$. Substrates were rotated during both depositions. For metals, 80W DC power was applied to a pure 2" target.

The $\mathrm{ZnO}$ layer and metal contact layers were patterned using $1 \mathrm{~cm}^{2}$ shadow masks (Suron Precision Technology). Shadow masking was used to avoid issues with photoresist at the high temperatures required $\left(300^{\circ} \mathrm{C}\right)$ for the $\mathrm{ZnO}$ deposition, as well as to avoid heating the $\mathrm{ZnO}$ layer during the photoresist process prior to the metal depositions. Additionally, shadow masking reduced the amount of sample loss during the lift-off process following deposition.

A common technique for patterning electrodes and other features on silicon chips is photolithography. In this technique, a pattern is transferred from a glass and chrome photomask to a polymer coating on a wafer using an instrument which exposes the mask and wafer to light. A polymer called a photoresist is applied to a wafer by a process called spin coating. In this process, the wafer is rotated, or spun, at high speeds while a photoresist is applied to the center of the wafer with a pipette. As the wafer spins, the applied droplet flattens out and coats the 
wafer surface in an even, uniform, coating. The photoresist is then baked for a short period of time to harden the polymer before the photomask is placed over the wafer. Aligning the mask to the wafer surface is typically performed using a mask aligner. The glass and metal (typically chrome) photomask is brought into close contact with the surface of the photoresist on the wafer, and the system is exposed to light, typically in the UV range. For the type of photoresist used, called a negative photoresist, the light cross-links the polymer. This is known as the 'exposure step'. Following the exposure step, the wafer is treated with a developer, which causes the regions exposed to light harden and remain after development, while dissolving the unexposed areas. This is usually followed by a metal deposition step, which results in metals depositing on both the wafer surface and the remaining photoresist. The final step, or 'lift off', dissolves the remaining photoresist, typically in acetone. Metals which deposited on the photoresist are washed off, while metals which deposited on the wafer surface remain.

An alternative approach to achieving metal patterns on a wafer is called 'shadow-masking' and uses a thin metal device called a shadow mask. This technique is much more straightforward, as the shadow mask simply acts as a stencil. The mask, which is a thin piece of metal with the pattern etched or cut out, is placed directly on top of the wafer while metal or other materials are deposited in a vacuum chamber. Shadow masking is preferred when a high-temperature deposition is required, but can only be used for larger features due to limitations in fabricating the metal shadow mask itself.

Two shadow mask designs were used to achieve the sample pattern shown in Fig.2. First, a mask with five vertical slits (Fig.2B) was clamped to a $1 \mathrm{~cm} 2 \mathrm{Si} / \mathrm{SiO} 2$ prepared substrate. Following $\mathrm{ZnO}$ deposition the substrates were removed, inspected visually and with optical microscopy, and the masks were replaced. The second set of mask patterned the electrical 
contacts (Fig.2C). Mask position was inspected with optical microscopy, and then sputtered with an adhesion layer of aluminum followed by a thicker layer of platinum.
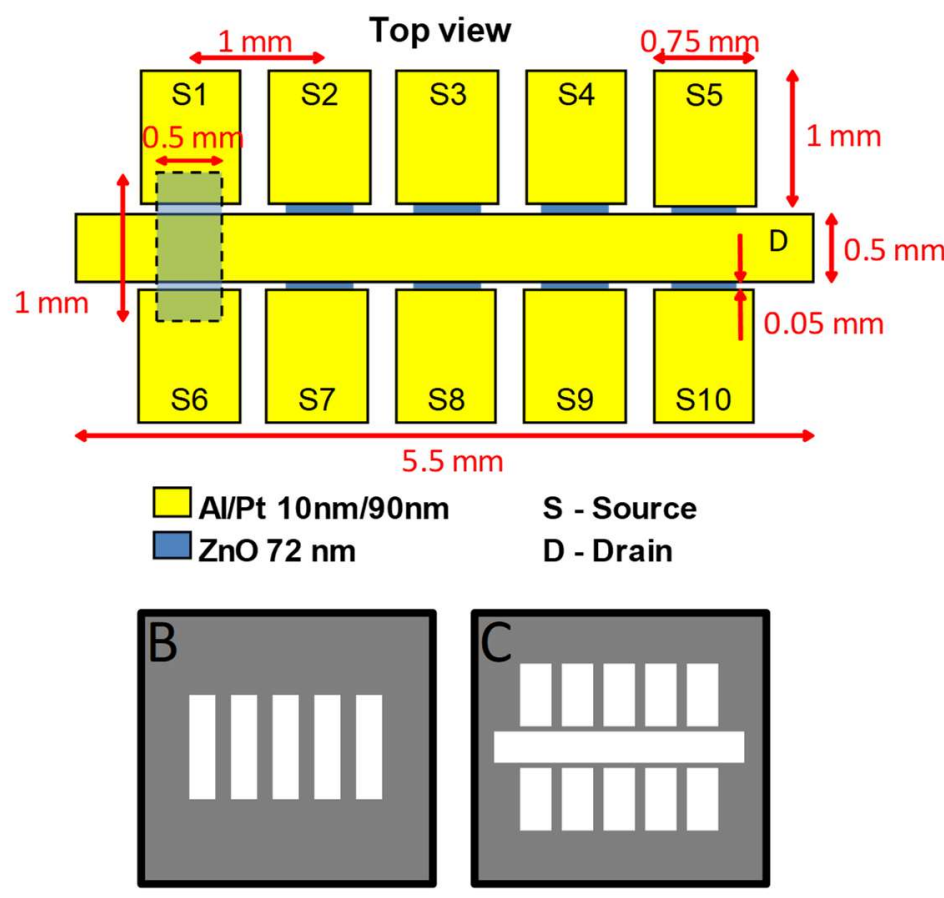

Figure 2. FET pattern using shadow masks.

Blue vertical bars represent $\mathrm{ZnO}(72 \mathrm{~nm})$, yellow represents $\mathrm{Al} / \mathrm{Pt}$ contacts $(10 \mathrm{~nm} / 90 \mathrm{~nm})$. This design featured a central horizontal contact which served as a drain (D), as well as ten source contacts (S1-10). The semiconducting channel in this device was $500 \mu \mathrm{m} \times 50 \mu \mathrm{m}$, with the gate contact on the back of the device. The masks $\mathbf{B}$ and $\mathbf{C}$ were used to pattern the Zinc Oxide (blue, B) and electrical contacts (yellow, C) respectively.
For many preliminary fabrication attempts, metal contacts were patterned using photolithography. AZ5214 photoresist (Microchem) was applied to wafers using a Laurel Technologies 400 spinner.

Photomasks were custom fabricated (Advance Reproductions Inc) to form the pattern shown in Fig. 3. Pattern alignment and UV exposure were performed with a Suss Microtech MA6 mask aligner using a 320 nm UV exposure source. Pattern development was performed using MIF 300 Developer (Microchem) according to the manufacturer's

instructions. Contact materials were $\mathrm{Ti} / \mathrm{Au}, \mathrm{Al} / \mathrm{Pt}$, or $\mathrm{Al} / \mathrm{Ti} / \mathrm{Au}$ depending on the sample, and were deposited using a Temescal BJD 2000 E-Beam evaporator. A number of contacts were also fabricated using a CVC 610 DC Magnetron sputtering station. Lift-off was performed in acetone. Initial parameters for the photolithography process were provided by Microchem, Inc., and were further adjusted based on thickness results obtained using a step profilometer (Tencor AlphaStep 200). 


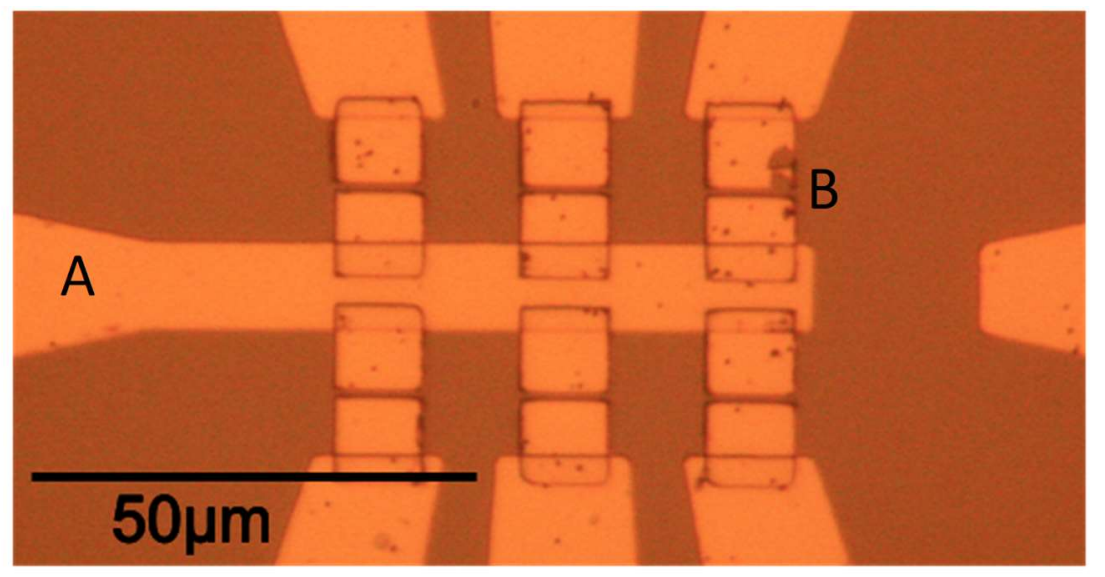

Figure 3. Photolithography and E-beam Lithography.

The above pattern was created using a combination of photolithography (A) and e-beam lithography (B). This pattern is amenable to functionalization with carbon nanotubes because the gap distance between the source and drain electrodes is $\sim 1 \mu \mathrm{m}$.
Subsequently, ZnO FETs with small (1um) channel width were fabricated with electronbeam (E-beam) lithography (Fig 3B). PMMA 950 A-9 (Microchem) was used as a photoresist, and was selected because of its thickness, which was desirable for the lift-off process and good contact between the small and large patterned metal contacts. Lithography was performed using a Leica 360 SEM with Nanometer Pattern Generation System (NPGS) software (Nabity 2018).

E-beam evaporation is a technique for depositing materials, usually metals, on a substrate. This technique was desirable because the thickness of the deposited layer was tightly controlled to within a few nanometers, and produced a uniform layer across the substrate. In addition, the deposition was directional, which means that material was only deposited on the surfaces perpendicular to the source. This was critical for small patterns and samples where sidewall deposition needed to be minimized.

In short, e-beam evaporation works by using an electron beam to heat a source material - in our case, a crucible filled with metal - causing it to vaporize and create a directional plume. This plume then coats the substrate, which hangs inverted over the source. This instrument had a quartz crystal monitor (King 1964), which is a device that measured the amount of deposited material based on shifts in the crystal's resonant frequency. When mass was added to the 
crystal, the resonant frequency shifted, allowing for the detection of very small amounts of deposited material. By placing this monitor near the substrate, it was possible to accurately measure the amount of deposited material to within a few angstroms. In the present work, this technique was used to produce $\mathrm{Ti} / \mathrm{Au}$ and $\mathrm{Al} / \mathrm{Pt}$ films with tightly controlled thickness.

The devices created using a combination of photolithography, electron beam lithography, and electron beam evaporation (Fig. 3) were produced in an effort to make a carbon nanotube FET. This was not achieved in the course of this work.

To examine the crystallinity and thickness of sputtered ZnO films, we used XRD and XRR (Rigaku RU-300). Briefly, XRD and XRR work by using a monochromatic beam of X-rays to examine the crystal structure of a sample. The small wavelength of these x-rays allows them to partially penetrate the crystal lattice of the sample, interact with the electron density of the atoms within the crystal, and reflect or diffract off of the sample at measurable angles (Suryanarayana and Norton 1998).

To measure thickness, we use the principle of Snell's Law to compare the angles at which constructive and destructive interference occur, much like examining the thickness of an oil film on top of water. This allowed precise measurement of the $\mathrm{ZnO}$ layers thickness on top of the Si/SiO2 substrates (Huang et al., 1993).

For crystallinity measurements, the diffraction of the x-rays was measured at a range of angles and compared to literature values. The angles at which peaks in the intensity of the signal are detected are well characterized for most materials, allowing us to confirm the crystal structure of our sputtered $\mathrm{ZnO}$ films. Films deposited with a substrate temperature below $250^{\circ} \mathrm{C}$ exhibited a zinc blende structure, while films deposited at $300^{\circ} \mathrm{C}$ showed a wurtzite structure, indicated by the peak near 34 (Fig. 1F) (Suryanarayana and Norton 1998; Pan et al., 2001). 
AFM was used in this work to characterize the roughness of sputtered $\mathrm{ZnO}$ films, as well as to examine the channel geometry for the narrow-channel devices. Briefly, AFM works by tapping or dragging a cantilever over the surface of a sample. In tapping mode, the cantilever is oscillated at a resonant frequency and brought close to the sample surface. As the tip of the cantilever approaches the surface, van der Waals forces between the tip and the sample change the resonant frequency of the cantilever. By scanning across a sample in this manner, a map of the forces is made, and converted into a height map of the sample (Meyer 1992).

SEM is a common method for examining sample geometry and quality. Due to the wavelength of visible light (around $390 \mathrm{~nm}-700 \mathrm{~nm}$ ) there is a physical limit to the resolution of an optical microscope (Around $1.5 \mu \mathrm{m}$ ). This can be overcome by using accelerated electrons, which have a much smaller wavelength, allowing the resolution limit to be drastically lowered. For the work presented here, typical SEM observations were made with good resolution below $250 \mathrm{~nm}$. The optical limit is the same reason that electron beam lithography was required to make patterns with features smaller than $5 \mu \mathrm{m}$, while UV light was used for patterns larger than $10 \mu \mathrm{m}$. Thus by analogy, the two types of microscopy are similar with the exception that electron optics use 'lenses' that are made from electromagnetic coils instead of glass.

To measure the semiconducting properties of the $\mathrm{ZnO}$ films, we used the Hall measurement technique, which allowed us to determine the carrier concentration and mobility of the films. (Gajurel et al., 2016) This mobility refers to the ability of charge carriers (electrons or holes) to move through the material, which correlates to how well the material conducts electricity. The principle of the Hall technique is the Hall Effect, in which a transverse magnetic field is applied to the sample while the current and voltage are measured in the plane of the sample (Bayraktaroglu and Leedy 2008). 
Once the devices were patterned as FETs, we measured the performance of the FETs using two regimes - transfer and output (Bayraktaroglu and Leedy 2008). In a transistor, transfer refers to the measurement of the source-drain current at a fixed source-drain voltage at a range of gate voltages. For these measurements, we typically held the source-drain voltage at $20 \mathrm{~V}$ and swept the gate voltage from -50 to $50 \mathrm{~V}$ in increments of $1 \mathrm{~V}$. Output measurements record the source-drain current at constant a constant gate voltage while changing the source-drain voltage. These measurements were usually performed at a gate voltage of $50 \mathrm{~V}$, with sourcedrain voltage ranging from -20 to 20 volts, in $0.5 \mathrm{~V}$ increments.

\section{B. Aptamer fabrication and characterization}

Aptamer selection and characterization was carried out by our colleagues, Jessica Lear and Dr. Letha Sooter, who provided us with the sequence information and extensive guidance on the use of such aptamers. The SELEX process began with a library or pool of ssDNA oligonucleotides containing a randomized sequence of nucleotides flanked by PCR primers. In SELEX experiments, the randomized region was typically $20-40 \mathrm{bp}$ long with a diversity of up to $10^{15}$ different sequences (Klug and Famulok 1994). Diversity in the range of $10^{13}$ sequences was not uncommon - 22 randomized base pairs, for example, produce $4^{22}$ different sequences, or $\sim 1.76 \times 10^{13}$. These randomized regions were flanked by primers, which facilitated PCR. These primers were sequences of DNA that allowed the copying machinery - TAQ Polymerase - to bind to and copy the sequence. These oligonucleotide constructs were obtained commercially (Eurofins MWG Operon, LLC; Integrated DNA Technologies, Inc.).

Most of these oligonucleotides did not bind to the target of interest, so several rounds of selection, and purification and amplification were required. Typically, a target molecule was immobilized on a matrix or substrate, and the oligonucleotide library incubated for some period 
of time with the target molecule. Following this incubation, the immobilized target molecule was removed, and with it any oligonucleotides with an affinity for the target. The bound oligonucleotides were dissociated from the target and amplified.

Often, a selection was performed with a negative or off-target as well, so that sequences which bind to similarly structured molecules were removed from the pool (Klug and Famulok 1994). Removing sequences which bound to the off-target in principle should have helped to ensure that the final product of the selection was specific only to the target molecule, without interactions from other, sometimes structurally similar, molecules. It was, however, impossible to expose the putative aptamer pool to every possible off-target, so assumptions were made which account for the final application of the aptamer itself. It is prudent to include rounds of negative selection for contaminants that are expected to be in the test media.

There were numerous methods for achieving this separation, such as an immobilized matrix (Sassanfar and Szostak 1993), magnetic beads (Qian et al., 2009), and others (Stoltenburg et al., 2005). Capillary Electrophoresis SELEX, or CE-SELEX, is a variation of the SELEX process implemented here, which eliminated the need for a substrate or matrix, and was useful for small molecule targets that are difficult to immobilize without causing significant structural changes. This was necessary for cadaverine and MEK, which are small, biogenic amines.

Like gel electrophoresis, CE uses an electrical potential to move charged molecules from one end of a capillary to the other (Kemp 1998). Separation was achieved based on the ratio of the charge of the molecule to its resistance to flow, or hydrodynamic radius. Since all of the DNA aptamers had the same charge, they were all pulled through the capillary with the same force. Aptamers which were bound to a target molecule were physically larger, and had a greater resistance to flow than free aptamers. Unbound target molecules had less charge than ssDNA, 
and experienced less electrical force through the capillary. Due to the separation of these three species in time, it was possible to collect only the oligonucleotide-target constructs, so that selections could be performed without the need to immobilize the target.

After performing selections against MEK and cadaverine, the remaining aptamer pool was sequenced. Consensus sequences were identified, tested, and verified. From among these sequences, several were chosen for further study and characterization. Finally, one sequence for each compound was identified as the putative aptamer. The resulting single sequences for the cadaverine aptamer and the MEK aptamer were synthesized (Integrated DNA Technologies, Inc.) and stored for later use. Results for the aptamer selection process are not shown here, as this work was carried out by colleagues.

\section{C. FET functionalization}

Aptamer attachment was achieved via a silane linker, (3-glycidoxypropyl) dimethylethoxysilane (Gelest Inc.), which bound to the thiol group attached to the aptamers (Hagen et al., 2010). In practice this occurred as a two-step process. First, the silane was applied by incubating the FET in a $2 \%$ (wt) solution of dimethylethoxysilane in $95 \%$ (v) ethanol, and then heated to $100^{\circ} \mathrm{C}$ in a vacuum. Aptamers were diluted to $12 \mathrm{ng} / \mu \mathrm{l}$ and treated with a thermal shock folding step prior to attachment. To ensure that the aptamers were folded into the correct tertiary structure, this approach first heated aptamers to $95^{\circ} \mathrm{C}$ for 5 minutes, and then shock cooled them in ice water. Following this, the now folded aptamers were dispensed directly onto the active channels of the FET using a Hamilton syringe, positioned using a micromanipulator. Aliquots between 200 and $400 \mathrm{~nL}$ were used, to control the quantity of aptamers deposited on each channel. Using this method, we were able to estimate that between 1.6-3.0 $\times 10^{11}$ aptamers were deposited on each channel. A rough geometric calculation accounting for the approximate radius of the aptamer 
$(\sim 75 \AA)$ and the size of the channel $(50 \mu \mathrm{m} \times 500 \mu \mathrm{m})$ predicted that this was more than enough aptamers to cover the surface.

Electrical measurements comparable to those made on untreated FETs were performed on functionalized FETs. Following our observation that illuminating the device caused a reduction in trapped charges (Gajurel et al., 2016), each measurement was preceded by 120 seconds of optical illumination by a fluorescent lamp. Transfer and output measurements were performed exactly as described above. For our measurements of functionalized FETs, it became necessary to measure the current as a function of time. For these measurements, the source current (l $\mathrm{DS})$ was measured with $10 \mathrm{~V} \mathrm{~V}_{\mathrm{G}}$ and $-20 \mathrm{~V} \mathrm{~V}_{\mathrm{DS}}$

\section{D. Functional Apta-FET testing}

Functional testing the Apta-FET devices required extensive measurements before and after each application of analyte. An 8 log-step concentration curve was prepared by serial dilution and presented to the device ( $1 \mathrm{pM}$ to $10 \mu \mathrm{M})$. Each concentration point was applied as an aliquot of $200-400 \mathrm{~nL}$, allowed to incubate for 5 minutes, and then briefly rinsed with $1 \mathrm{~mL} \mathrm{DI}$ $\mathrm{H}_{2} \mathrm{O}$ and dried with $\mathrm{N}_{2}$. The brief rinse step mitigated spurious effects due to evaporation of the $\sim 400 \mathrm{~nL}$ aliquot, and the $\mathrm{N}_{2}$ drying reduced damage to the semiconducting channel. After measuring $I_{D S}$ as a function of time for $120-240$ s on each channel as described above, the device was incubated in $\mathrm{DI} \mathrm{H}_{2} \mathrm{O}$ and rinsed with a pipette for 5 minutes. Electrical measurements were taken after rinsing to allow for baseline comparison. It became evident after the first several tests that the device did not return to baseline after exposure to analyte; measuring the steady-state current after rinsing helped account for this behavior. 
For the final phase of testing, we calculated the normalized current at steady-state. This typically consisted of the measured source-drain current between 100 - 120 seconds after the voltage was applied $(n=30-40)$. This current was then normalized as $I / I_{0}$, with $I_{0}$ being the steady state current observed after a blank $\left(\mathrm{DI} \mathrm{H} \mathrm{H}_{2} \mathrm{O}\right)$ measurement.

Our preliminary data showed that after rinsing, there was a substantial persistent effect on gate voltage left over from the previous trial. After each log step in concentration, we rinsed the chip, and the signal did not return to the baseline recorded prior to beginning the measurement series. To account for this when analyzing the data, we subtracted the signal from the prior rinse step. For example, when calculating the normalized current $\left(\mathrm{I} / \mathrm{I}_{0}\right)$ for a $1 \mu \mathrm{M}$ treatment, we subtracted the normalized current from the rinse step after the 100nM treatment.

In an effort to improve selectivity, we incubated a functionalized Apta-FET with bovine serum albumin (BSA). This was done to address a suspected issue with nonspecific binding of the analytes to the chip surface or ethoxysilane linker layer. To achieve this, Apta-FET 20160513B was incubated in a $2 \%$ solution of BSA in phosphate-buffered saline for 30 minutes at room temperature, and then rinsed with DI water. The chip was then dried with $\mathrm{N}_{2}$ and mounted on a test platform for later use. Although this appeared to increase the selectivity of the device, the results were unclear, and the aptamer without BSA showed similar response to the BSA without aptamer, suggesting that the BSA may contribute to analyte binding in a preferential manner. Thus, while it appeared to increase the selectivity, this is likely due to an increase in specific binding of BSA to an analyte, as opposed to a reduction of nonspecific binding. 


\section{Results}

\section{A. Non-functionalized FETs}

Each FET was tested for its electrical performance. Output and transfer curves, as well as a measurement of current over time, were obtained immediately after device fabrication (Fig. 4). The transfer and output plots shown here are representative of a good, working FET. At large, negative gate voltages, the FET is effectively switched off, inhibiting current flow. As the gate voltage approaches the threshold voltage $V_{T}$, the FET begins to switch on and allow current to flow. For the $\mathrm{ZnO}$ thin film FETs produced, positive gate voltage $\left(50 \mathrm{~V}_{\mathrm{G}}\right)$ and negative drainsource voltage (-20 VDS) allowed for the largest current flow lDs. Transfer plots are shown as loglinear due to the range of IDS. The leakage current was measured between the gate and the source. This current should be nonexistent due to the insulator between the gate, and the source and drain contacts. This current was measured for quality validation, and is shown as a dashed line.

\section{B. Functionalized FETs}

Preliminary trials with a functionalized FET (ZnO20150506B) showed that the transfer response (Fig. 5) decreased when the chip was exposed to the target analyte with the decrease being concentration dependent. This result was promising, but repeated transfer measurements were not feasible. In addition, it became apparent that the speed at which the measurement was acquired changed the amount of current measured, indicating that there was a time-dependent effect in the device. To obtain more accurate measurements, we chose to measure at a fixed gate voltage $\left(V_{G}=10 \mathrm{~V}\right)$ and drain-source voltage $\left(V_{D S}=-20 \mathrm{~V}\right)$. 


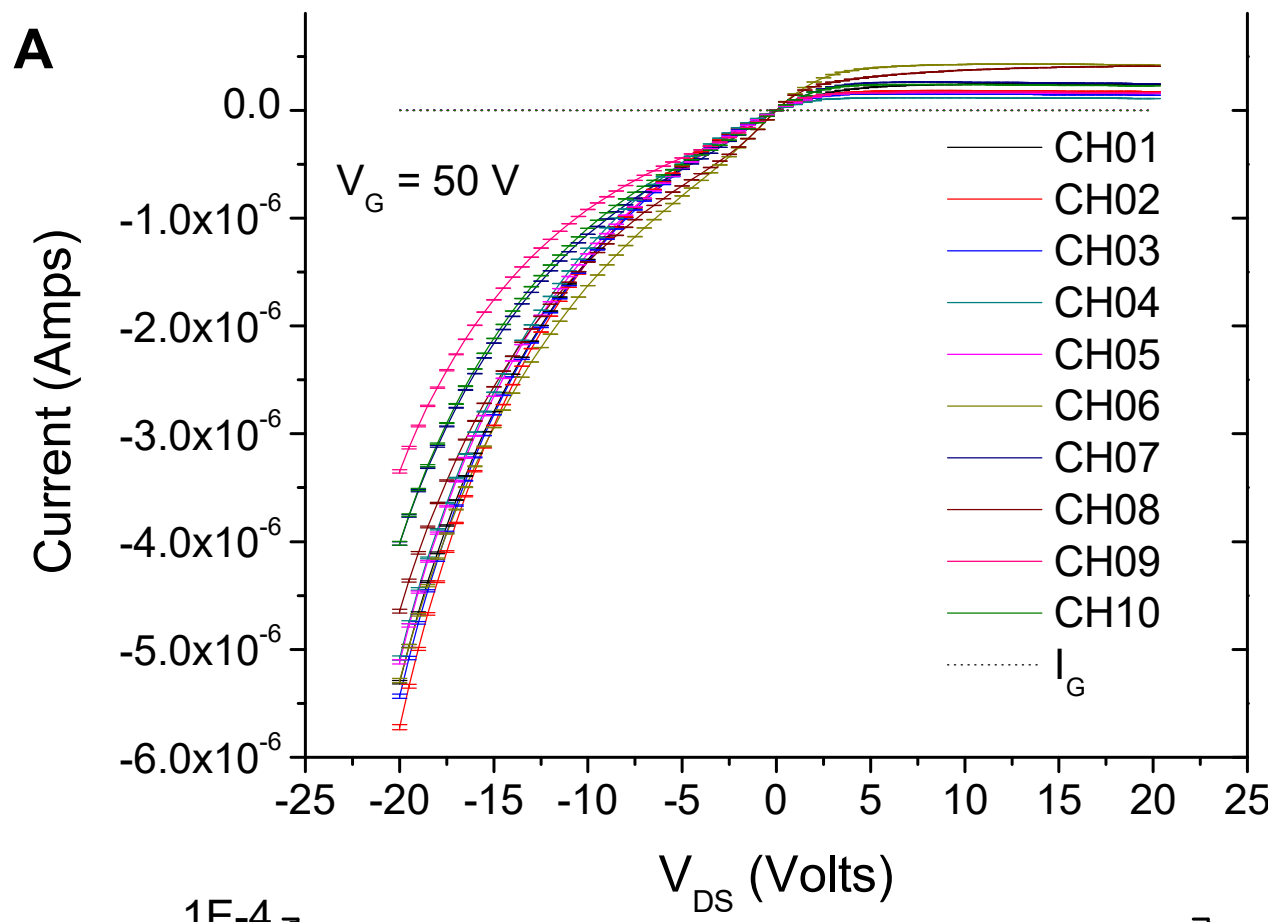

B

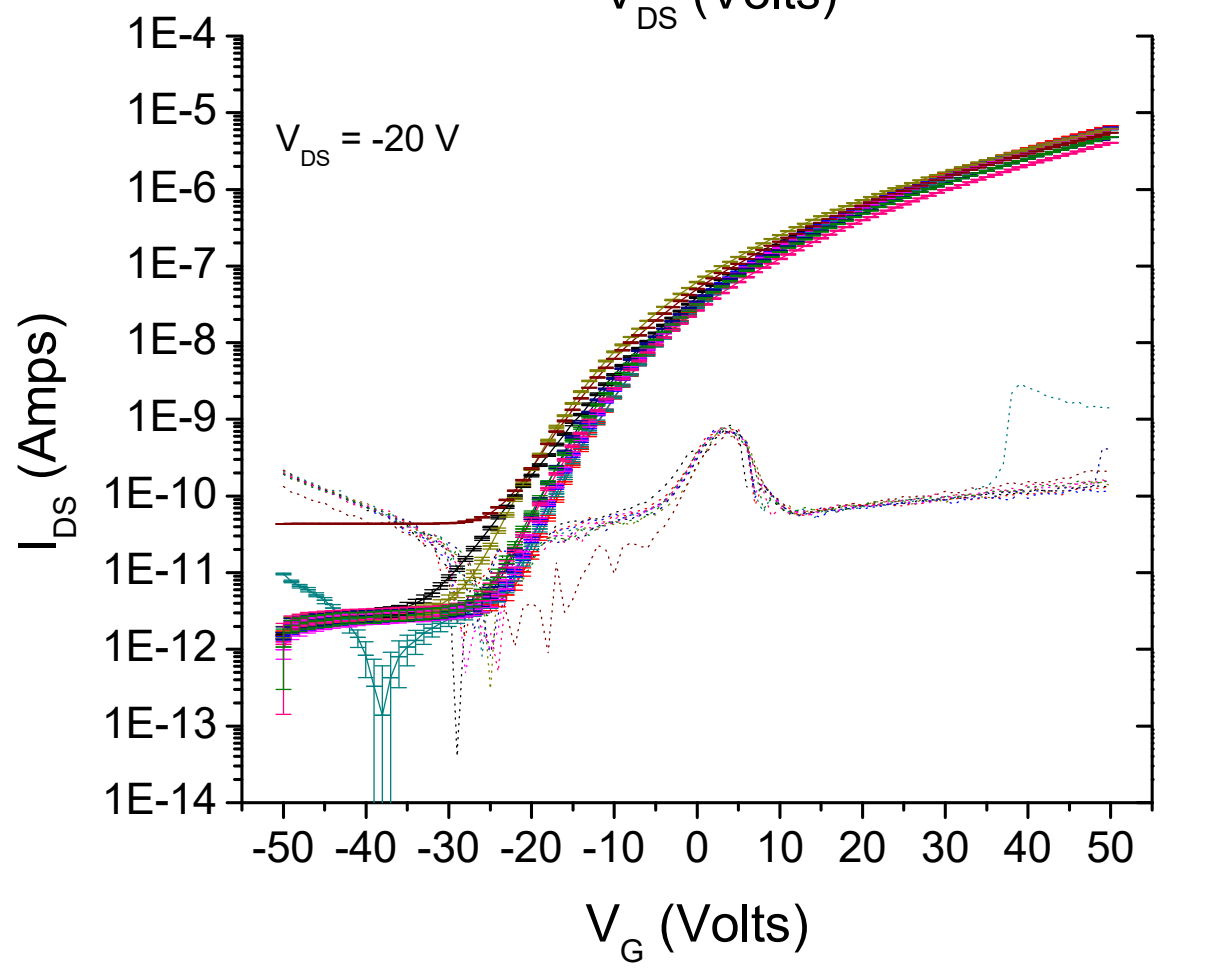

Figure 4. Output (A) and transfer (B) measurements.

FETs were measured immediately after fabrication. Output (A) was measured as the current between the source and drain (IDS) at different drain-source voltages $\left(V_{D S}\right)$ with a constant gate voltage $\left(V_{G}\right)$ of $50 \mathrm{~V}$. Transfer (B) measurements measured $I_{D S}$ as a function of $V_{G}$ at a fixed $V_{D S}$ of $-20 \mathrm{~V}$. Data is shown for each of 10 channels on the same FET. The dotted lines indicate the leakage current, between the gate and the drain. Leakage current was measured to assess the quality of the insulation between the gate and the source / drain electrodes. 
Functional testing was performed (Fig. 6) by exposing the chip to increasing concentrations of the target or an off-target. These exposures consisted of applying an aliquot $(400 \mathrm{~nL})$ of analyte in water to the active channel of the chip, preceded and followed by a $\mathrm{DI} \mathrm{H}_{2} \mathrm{O}$ rinse to remove excess or unbound material. Following the second rinse, the chip was dried with UHP $N_{2}$. After each exposure, IDS was measured over time with $V_{D S}=-20 \mathrm{~V}$ and $V_{G}=10 \mathrm{~V}$.

Tests were performed with FETs functionalized with either ATP (ZnO20160503A) or cadaverine (ZnO20160503B) aptamers. Of the ten channels on the chip, the end and center channels were tested with analytes (ATP, MEK, and cadaverine) as described in Fig. 7. This eliminated the effect of analyte flowing from one channel to another and facilitated the application of controlled aliquots of analyte to specific channels. As demonstrated in Fig. 8, the response to increasing log steps in concentration produces a linear response in $1 / \mathrm{I}_{0}$. However, this response is also present for non-target analytes.
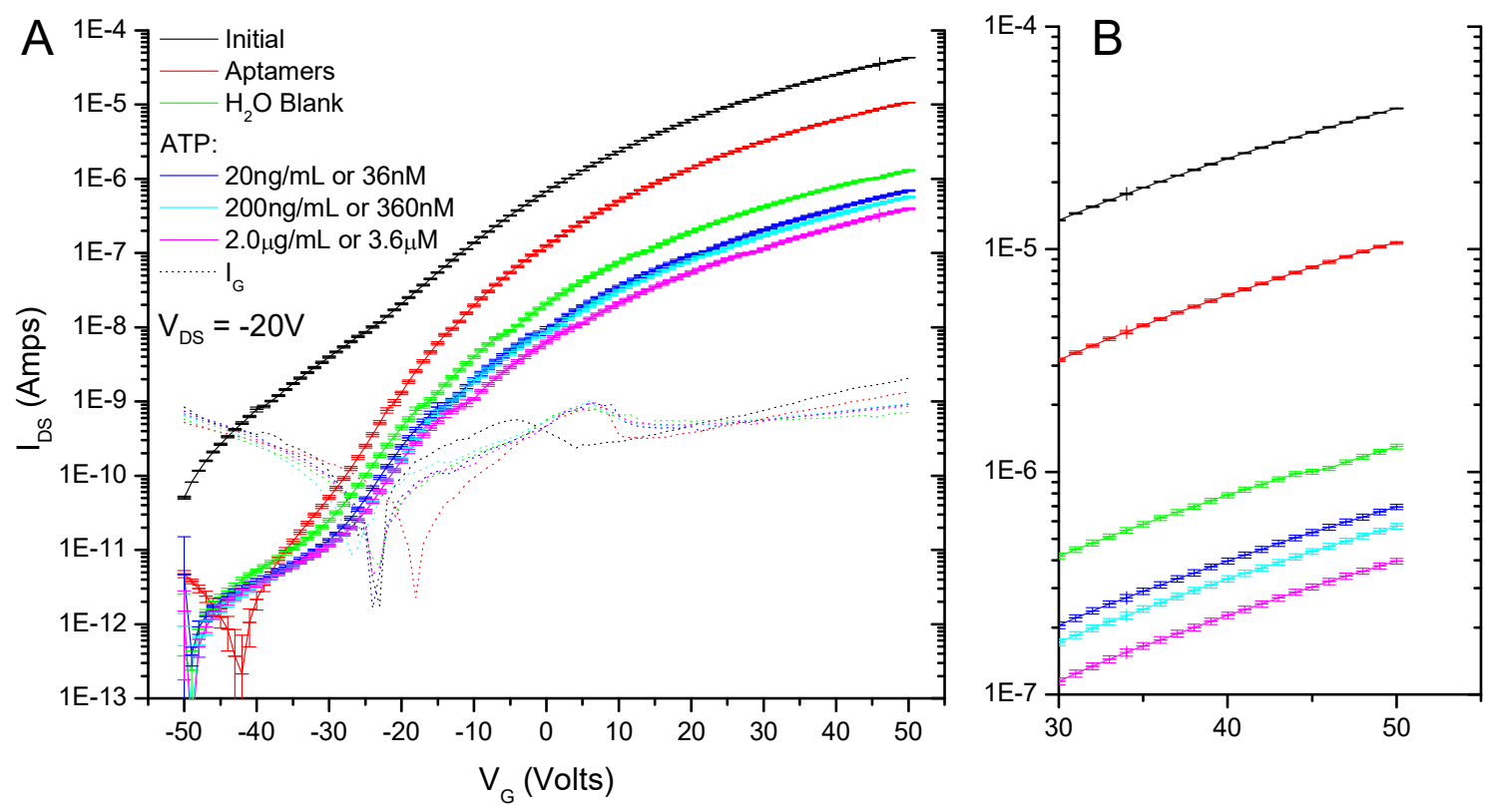

Figure 5. Preliminary electrical measurements of an Apta-FET.

A.) Transfer characteristics for a channel on FET-ZnO20150506B. Curves shown are prior to functionalization (initial, black line), and after functionalization with ATP aptamers (red line), with the addition and removal of water (green line). The Apta-FET was exposed to varying concentrations of ATP (blue, cyan, and magenta lines). Each increasing concentration shows a decrease in measured current. Dotted lines show the leakage current between the gate and drain. B.) Data from A.), rescaled for clarity. 
In an effort to improve selectivity, we treated a FET (ZnO20160513B) with BSA. This should have reduced non-specific binding of the analyte to the silane linker layer if it were a source of problems with selectivity. To investigate this, several channels on the same Apta-FET received different treatments (Fig. 9). The entire chip was treated with the
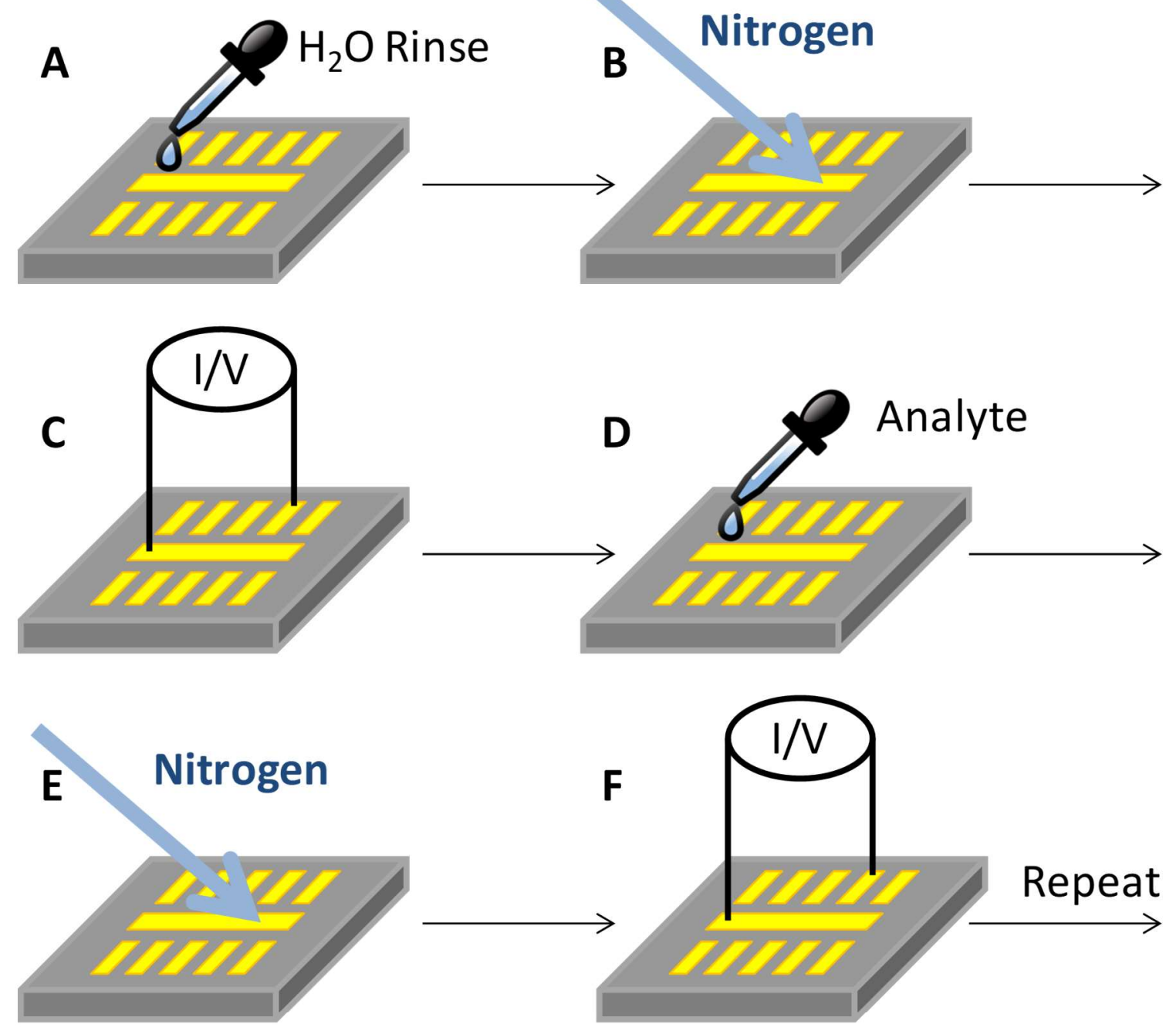

Figure 6. Functional testing procedure.

Apta-FETs were tested by applying $\sim 0.2 \mu \mathrm{L}$ of liquid analyte, delivered by a syringe. (A) Chips were rinsed with $18.2 \mathrm{M} \Omega \cdot \mathrm{cm} \mathrm{H}_{2} \mathrm{O}$ and (B) dried with UHP $\mathrm{N}_{2}$. (C) Electrical measurements were obtained after each rinsing / drying cycle. (D) Liquid analyte was delivered to the Apta-FET, and then $(E)$ dried with with UHP $N_{2}$. (F) Electrical measurements were obtained again. This process (A-E) was repeated for each log-step increase in analyte tested. 


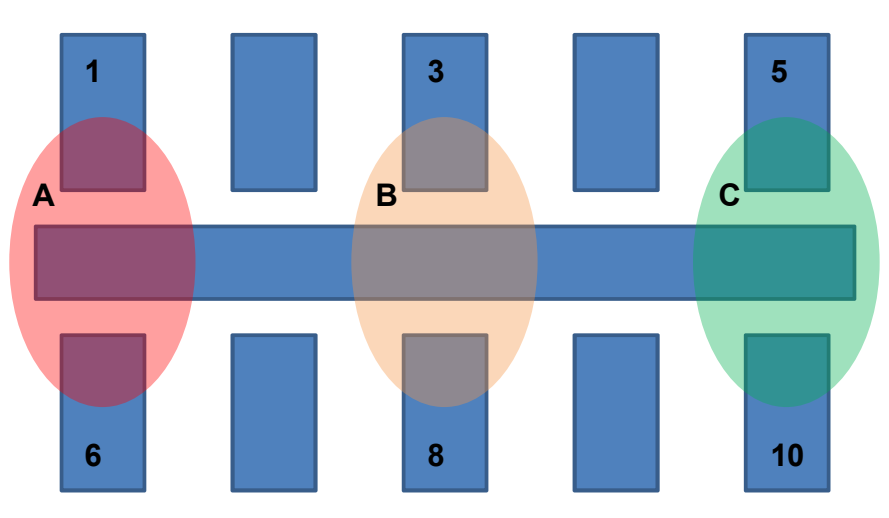

Figure 7. Functional testing paradigm for multi-analyte testing.

Aliquots $(200-400 \mathrm{~nL})$ at different concentrations of ATP (A, red), cadaverine (B, orange), or MEK (C, green) was applied to channels. dimethylethoxysilane linker layer in the

same manner as other Apta-FETs.

Channels 7-9 were functionalized with the

ATP aptamer, channels 8-10 were treated

with BSA. This resulted in four types of

channels. Channel 5 was treated with

silanes only, channel 7 was treated with the

ATP aptamer on silanes, channel 10 was

treated with BSA on silanes, and channels

8 and 9 were treated with silanes, ATP

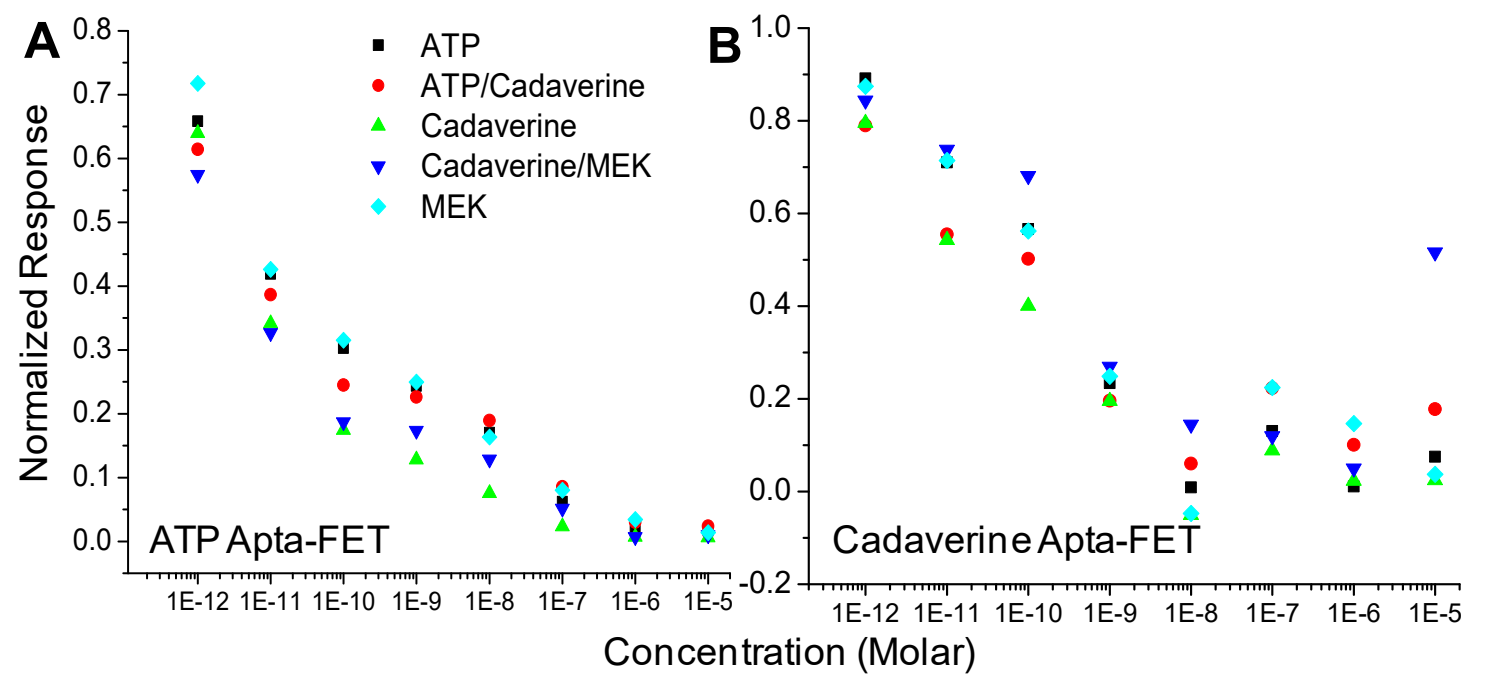

Figure 8. Response of Apta-FETs ZnO20160503A and ZnO20160503B.

Apta-FETs were tested with three different analytes after being functionalized with either ATP aptamers (A) or cadaverine aptamers (B). Channels 1 and 6 were exposed to ATP (black squares); channels 3 and 8 were exposed to cadaverine (green triangles); channels 5 and 10 were exposed to MEK (teal diamonds). "Buffer channels," $(2,4,7,9)$ were exposed to two analytes as a result of the testing method, and are shown as red circles (ATP/cadaverine) and blue triangles (cadaverine/MEK). Averaged responses from channel pairs with the same analyte are shown. Both chips show concentration-dependent responses to all analytes. Normalized response is a function of normalized current, $1 / I_{0}\left(I_{0}\right.$ is defined by the response to $\left.\mathrm{DI} \mathrm{H} O\right)$. 
aptamer, and BSA. Channels 1-4

and 6 were not used. To obtain the

experimental results shown in Fig.

9 , channels $5,7,8$, and 10 were

treated with ATP, while channel 9

was treated with cadaverine. This

allowed us to compare two identical

channels (8 and 9) tested with the

target (8) and off-target (9). In

addition, we were able to show the

effect of adding BSA on the

sensitivity of the ATP-aptamer

functionalized channels when

presented with the target (ATP,

compare channels 7 and 10).

Furthermore, since channel 5 was

treated only with silanes, but not

aptamers, it shows the contribution

of non-specific binding in the

response. As shown in Fig. 9, the

addition of BSA to channels treated
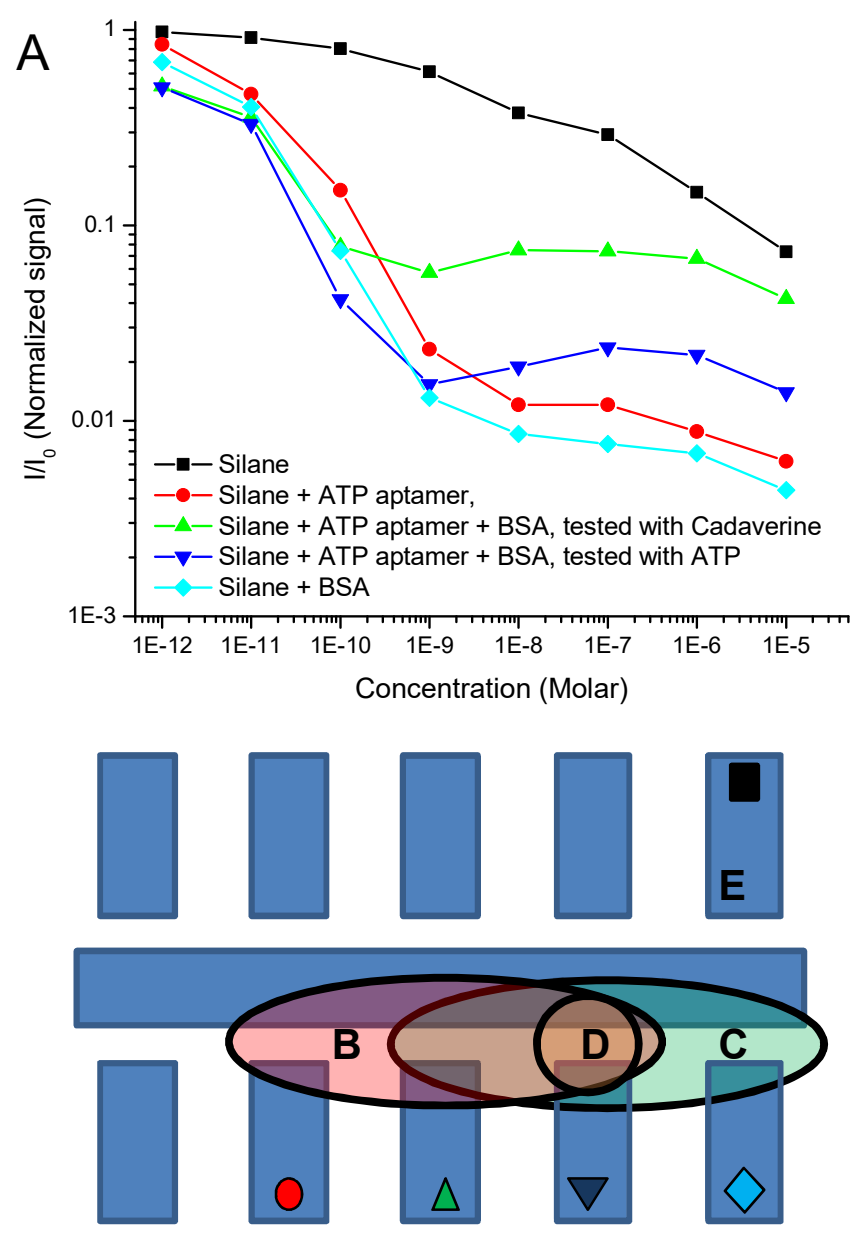

Figure 9. Effects of blocking with BSA.

A.) Measurements performed on Apta-FET ZnO20160513B. All channels were initially treated with dimethylethoxysilane (linker molecule). Symbol markers correspond to channel numbers in both figures: $5 \bullet \mathbf{\bullet} 7 \bullet, 8 \Delta, 9 \boldsymbol{\nabla}, 10$ \&. B.) Channels 7,8 , and 9 were functionalized with the ATP aptamer (red ellipse). C.) Channels 8,9 , and 10 were blocked with BSA (green ellipse). D.) All channels were tested using ATP, except for channel 9, which was tested with cadaverine (orange circle). E.) Channel 5 was left unmodified as a reference.

with ATP aptamers resulted in an increased discrimination between the target, ATP (down triangles), and the off-target, cadaverine (up triangles). Responses from the BSA-blocked channels with ATP aptamer (down triangles) and without ATP aptamer (diamonds) indicates that BSA itself is contributing to the signal at unacceptable levels, nearly comparable to the channel functionalized with aptamer but without BSA (circles). 


\section{Discussion}

We successfully fabricated a ZnO FET functionalized with three distinct aptamers, selected for cadaverine, MEK, and ATP. The ZnO FET exhibited a measurable change in performance between -50 and $50 V_{G}$, with the strongest sensitivity to gate voltage at $+20 V_{D S}$. The Apta-FET device demonstrated electrical sensitivity to picomolar concentrations of analyte, although discriminatory power was poor.

\section{A. FET Performance - Optical Resetting}

Additionally, we showed that our FET responded to illumination at different wavelengths by resetting the charging effect of the gate. Gate charging is an undesirable effect in field effect transistors (Gajurel et al., 2016), and our work describes optical resetting of the device as a potential remedy. Gate charging occurs when charges remain at the gate-insulator interface after the gate voltage is removed, and results in loss of FET response after subsequent gating cycles. We eliminated this problem by exposing the device to illumination (Gajurel et al., 2016).

\section{B. Sensitive, But Not Selective}

The aptamer-decorated FET that we produced (Apta-FET) was able to detect the addition of analyte at picomolar $(\mathrm{pM})$ concentrations, and continued to show incremental and measurable changes in response up to concentrations as high as 10 micromolar $(\mu \mathrm{M})$. However, the device was very limited in its selectivity between analytes; our work was unable to show conclusive discriminatory power between exposure to the target and the off-target. Nonetheless, our work demonstrates that the aptamers attached to the surface of the device were able to modulate the function of transistor. 
Additionally, when compared to other methods, our device was able to detect very low concentrations of analyte. A similar device consisting of aptamer-functionalized graphene achieved detection of a 140 nanomolar (nM) solution of posaconazole, and discrimination between posaconazole and capsofungin at $\sim 140 \mu \mathrm{M}$ concentration (Wiedman et al., 2017).

The NIOSH (National Institute of Occupational Safety and Health) Manual of Analytical Methods (NMAM) method number 2500 for detecting MEK in air describes a technique for sampling air with a sorbent tube, extracting the sampled MEK, and quantifying the amount present using gas chromatography / mass spectrometry. The limit of detection specified is $0.004 \mathrm{mg} / \mathrm{sample}$, which equates to $\sim 55.5 \mu \mathrm{M}$ MEK in extraction solvent. Although it is likely possible to detect lower concentrations of MEK using GCMS, NMAM 2500 provides a good benchmark as an accepted standard method for quantifying concentrations of MEK in air (NMAM 2500).

At the lower limit, liquid chromatography / mass spectrometry (LCMS) can be used to detect trace amounts of analyte. Ibarra et al., published a method for detecting cadaverine and other small amines in 2015 , with a quantification limit of $\sim 1.3 \mathrm{nM}$ and detection limit of $\sim 0.4 \mathrm{nM}$ for cadaverine (Ibarra et al., 2015). This is still ten times more concentrated than the detection limit achieved with the Apta-FET. Thus while the selectivity for the target analyte was lacking, the sensitivity is unprecedented in the literature.

\section{$\underline{\text { IV. C. Limitations and proposed solutions }}$}

While the Apta-FET we designed showed promising results for sensitive detection, our FET did not perform as well electrically as other devices in the literature. Better device fabrication could be achieved by reducing the thickness of the insulating oxide layer (currently 200nm). 
Additionally, numerous types of FETs exist, and many of them are amenable to aptamer attachment (Staii et al., 2005; Aliakbarinodehi et al., 2017).

A limitation of the work presented herein was our inability to expose our device to airborne or vapor samples of our test analytes. A major motivation for the design of the Apta-FET was to detect small molecules - odorants - in air. However, due to testing limitations, the device was only measured after exposure to liquid aliquots applied directly to the device. This constraint had three major disadvantages.

Primarily, we were unable to determine if the aptamers would be able to function in a nonaqueous environment. Altering the immediate molecular environment around the nucleic acid would, in theory, alter its folded structure (Feigon et al., 1996; Lin and Patel 1997). Additionally, it is assumed that water molecules facilitate the binding interactions between aptamer and target. One of the goals of the study was to determine how aptamers would perform in a relatively drier microenvironment.

Second, the addition of liquid water to the surface of the device greatly limited our available voltage parameters for functional testing. Typical performance tests for our FETs required -50 to $50 \mathrm{~V}_{\mathrm{G}}$ and -20 to $20 \mathrm{~V}_{\mathrm{DS}}$. However, due to the small distance between the source and drain $(50 \mu \mathrm{m})$, potentials higher than $2 \mathrm{~V}_{\mathrm{DS}}$ were impossible while aqueous solution was on the chip. In order to use the voltages required for an acceptable device response, we had to incubate the sensor channel in a small aliquot of target solution, and then allow the droplet to dry by evaporation. This introduced additional potential for spurious effects, such as dried analyte on the surface, hydrodynamic repositioning of aptamers, changes to aptamer folding, and residue from impurities deposited on the device surface. 
Our practice of applying a liquid droplet to the surface of the device and then allowing the droplet to dry limited our ability to accurately determine the concentration of analyte delivered. The volumes added were small, on the order of $200-400 \mathrm{~nL}$. The accuracy of the delivery was limited, both in position on the device and volume, despite the use of optical magnification and micromanipulator devices. This problem can be overcome in future testing by exposing the device to an air stream containing the volatile compounds of interest. Currently, such a system is in use for the characterization of similar devices at the National Institute of Occupational Safety and Health, Respiratory Health Division (NIOSH/RHD) in Morgantown, WV. By using this method, we will be better equipped to examine the response of the Apta-FET to prepared concentrations of target in air.

In order to complete the stated aims of the original work, the Apta-FET needs to be tested in the gas or vapor phase as well. While it is possible that the cross-reactivity issue may persist, evidence from Apta-FET trials indicates that the addition of liquid water droplets induce an electrical change in the device, even after the droplet has dried. The best way to verify this experimentally would be to measure the Apta-FET in a vapor environment.

In order to conclusively investigate the performance of the Apta-FET, measurements need to be taken in a dynamically generated flow cell. Such an experiment could be performed by flowing vapor containing the target over the device while measuring the current at a set $V_{G}$ and $V_{D S}$. This would clearly demonstrate if the Apta-FET is capable of detecting target analytes in air, and would allow a more direct investigation of cross-reactivity. In addition, it would inform us about the nature of the saturation issue, and give evidence about the role of liquid water in aptamer function. 
Due to the duration and logistics of the testing process, it is possible that the aptamers attached to the chip surface suffered physical damage during testing as a result of wetting and drying. Dust, while minimized, was present because the electrical measurements could not be performed inside a cleanroom.

A final limitation of the Apta-FET was that we were unable to reset the sensor to a baseline after the addition of analyte. In practice, our detection threshold for each test was determined by the highest concentration of analyte previously applied to the chip. This effect persisted after optically resetting the FET, after rinsing the device in $\mathrm{DI} \mathrm{H}_{2} \mathrm{O}$, and after allowing the chip to sit for a period of several weeks. Again, it is not clear if this is due to irreversible binding of the target to the aptamer, or other effects introduced by the application of a liquid sample.

There are several potential methods to mitigate this problem. One possibility is to denature the aptamers with heat or a strong electrical field; however, both of these could damage the FET. In addition, there is a risk that the aptamers may not return to their native conformation after being denatured. Another possibility is extensive washing or rinsing. While we did see some effect of rinsing the chip on subsequent baseline measures, it was not sufficient to reset the device. More work is needed to understand this limitation.

\section{D. Proposed Reasons for Nonspecific Binding}

The Apta-FET produced here, while sensitive to concentration, could not discriminate between analytes using the methods employed, that is, the selectivity of the Aptamers for the target appears to have been lost. This could be because the aptamers respond indiscriminately to the different analytes tested. It is possible that the aptamers, when attached to the chip and exposed to a dry environment, lose their conformation and thus their specificity. It is also 
possible, although unlikely, that the ATP aptamers respond equally to ATP, MEK, and cadaverine, and that the cadaverine aptamers respond in kind. A third possibility is that a component of the device, such as the silane attachment layer or the $\mathrm{ZnO}$ itself, reacts with the analytes tested to produce a confounding response. Attempts to ameliorate this possibility using BSA was met with limited but encouraging success.

In an attempt to reduce non-specific binding, we attempted to block the surface of the FET with bovine serum albumin (BSA). We reasoned that there may be sites on the surface of the device or on the functionalized layer that could interact nonspecifically with ATP, cadaverine, or MEK. This resulted in Apta-FETs with reduced cross-reactivity, but a lower overall response that was less dependent on concentration. A possible explanation for this is that since BSA itself is capable of binding to many different compounds, it may have acted as an intermediate layer, reducing the sensitivity.

Future efforts might focus on testing different concentrations of BSA applied to the functionalized chip, investigating the conformation of the aptamers attached to the chip, and investigating the binding ability of the attached aptamers to their target compounds. It might be possible to apply a test mixture containing the target to the chip, and later analyze the chip surface for the target. Such an experiment could be performed by exposing the Apta-FET to the target, incubating for some time, cleaving the aptamer-silane attachment point, and then using an analytical technique (such as liquid chromatography / mass spectrometry) to confirm the presence of the target.

A potential reason for the off-target responses of the Apta-FET could be that the surface-bound aptamers are not folded properly. In order to exhibit binding activity and selectivity, the aptamers need to be in the same configuration that they were in vitro when they were selected using the 
SELEX process (Ellington and Szostak 1992). Although the aptamers were folded by heating and shock cooling prior to attachment to the chip, it is possible that repeated cycles of wetting and drying during the functionalization process caused the aptamers to denature or adopt a different conformation. If this is the case, it would explain the general sensitivity of the chip to different compounds, since it is known that unfolded DNA can interact and bind nonspecifically to various compounds. This can be ameliorated in future work by negatively selecting for more off-target compounds during the aptamer selection process (Klug and Famulok 1994).

Another explanation for off-target response is that the aptamers used are cross reactive for the different analytes used. While we do not expect that the ATP aptamer can bind to MEK and cadaverine, we have not verified this in an independent experiment. Negative selections were not performed for the cadaverine aptamers to ensure that it does not bind to MEK. It is therefore possible, albeit unlikely, that there are responses to these three different analytes from all three aptamers because the aptamers themselves are capable of binding to each analyte.

We have also considered that the intermediate ethoxysilane linker layer could be binding nonspecifically to our analytes. The silane layer itself could trap or bind to our analytes, resulting in a change in electrical response from the Apta-FET. To investigate this further, we functionalized an FET with only the silane linker but did not add aptamers. In these tests, we did not see an electrical response comparable to the off-target interactions from the Apta-FET. This leads us to conclude that the silane layer was not solely responsible for the off-target interactions. 


\section{Conclusion}

The present work describes the fabrication and characterization of an electronic nose device consisting of a thin film zinc oxide field effect transistor functionalized with DNA aptamers. The goal of this work was to produce an electronic device which detects and reports the presence of small molecules using the principles of olfactory receptors. This device is capable of sensitive detection of small amounts of analyte, but has poor discriminatory power. Our work suggests that aptamers may not retain their specificity if dried and rehydrated, but further study is needed to confirm this. 


\section{REFERENCES}

Abdollahi A, Jiang Z, Arabshahi SA (2007) Evaluation on mass sensitivity of SAW sensors for different piezoelectric materials using finite-element analysis. IEEE Trans. 54:2446-2455.

Åbom AE, Comini E, Sberveglieri G, Hultman L, Eriksson M (2002) Thin oxide films as surface modifiers of MIS field effect gas sensors. Sens. Actuat. B: Chem. 85:109-119.

Ai M, Min w, Grosjean Y, Leblanc C, Bell R, Benton R, Suh GSB (2010) Acid sensing by the Drosophila olfactory system. Nature 468:691-696.

Aliakbarinodehi N, Jolly P, Bhalla N, Miodek A, De Micheli G, Estrela P, Carrara S (2017) Aptamer-based field-effect biosensor for tenofovir detection. Sci. Rep. 7:44409.

Alizadeh T, Zeynali S (2008) Electronic nose based on the polymer coated SAW sensors array for the warfare agent stimulants classification. Sens. Actuat. B: Chem. 129:412-423.

Bai H, Li C, Chen F, Shi G (2007) Aligned three-dimensional microstructures of conducting polymer composites. Polymer. 48:5259-5267.

Battiston FM, Ramseyer J-P, Lang HP, Baller MK, Gerber Ch, Gimzewski JK, Meyer E, Güntherodt H-J (2001) A chemical sensor based on a microfabricated cantilever array with simultaneous resonance-frequency and bending readout. Sens. Actuat. B: Chem. 77:122-131.

Bayraktaroglu B, Leedy K (2008) pulsed laser deposited ZnO for thin film transistor applications. ECS Trans. 16:61-73.

Bayraktaroglu B, Leedy K, Neidhard R (2008) Microwave ZnO thin-film transistors. IEEE Elect. Dev. Lett. 29:1024-1026.

Bear MF, Connors BW, Paradiso MA (2007) Neuroscience: Exploring the Brain 3rd ed. Baltimore, MD: Lippincott Williams and Wilkins.

Benton R, Vannice KS, Gomez-Diaz C, Vosshall LB (2009) Variant ionotropic glutamate receptors as chemosensory receptors in Drosophila. Cell 136:149-162.

Bedoui S, Faleh R, Samet H, Kachouri A (2013) Electronic nose system and principal component analysis technique for gases identification. IEEE 10th International MultiConferences on Systems, Signals \& Devices 2013 (SSD13).

Braik M, Dridi C, Ben Ali M, Ali M, Abbas M, Zabala M, Bausells J, Zine N, Jaffrezic-Renault N, and Errachid A (2015) Development of a capacitive chemical sensor based on Co(II)phthalocyanine acrylate-polymer/HfO2/SiO2/Si for detection of perchlorate. J. Sens. Sens. Syst. 4:17-23.

Brennan PA, Keverne EB (2004) Something in the air? New insights into mammalian pheromones. Curr. Biol. 14:R81-R89.

Briand L, Nespoulous C, Perez V, Rémy J-J, Huet J-C, Pernollet J-C (2000) Ligand-binding properties and structural characterization of a novel rat odorant-binding protein variant. Eur. J. Biochem. 267:3079-3089.

Buck L, Axel R (1991) A novel multigene family may encode odorant receptors: a molecular basis for odor recognition. Cell 65:175-187. 
Carcia PF, McLean RS, Reilly MH, Nunes G Jr (2003) Transparent ZnO thin-film transistor fabricated by RF magnetron sputtering. Appl. Phys. Lett. 82:1117.

Carew TJ (2000) Behavioral Neurobiology: The cellular organization of natural behavior. Sunderland, MA: Sinauer.

Chang AL, McKeague M, Liang JC, Smolke CD (2014) Kinetic and equilibrium binding characterization of aptamers to small molecules using a label-free, sensitive, and scalable platform. Anal Chem. 86:3273-3278.

Chen X, Wang Z, Duan N, Zhu G, Schwarz EM, Xie C (2018) Osteoblast-osteoclast interactions. Connect. Tissue Res. 59:99-107.

Daly KC, Carrell LA, Mwilaria E (2007) Detection versus perception: physiological and behavioral analysis of olfactory sensitivity in the moth (Manduca sexta). Behav. Neurosci. 121:794-807.

Daly KC, Wright GA, Smith BH (2004) Molecular features of odorants systematically influence slow temporal responses across clusters of coordinated antennal lobe units in the moth manduca sexta J. Neurophysiol. 92:236-254.

Daly KC, Bradley S, Chapman PD, Staudacher EM, Tiede R, Schachtner J (2016) Space takes time: Concentration dependent output codes from primary olfactory networks rapidly provide additional information at defined discrimination thresholds. Front. Cell. Neurosci. 9:515.

Daqi G, Zeping Y, Jianli S (2008) Modular neural networks for estimating odor concentrations. IEEE World Cong. Comput. Intel. 3940-3947.

Dear TN, Boehm T, Keverne EB, Rabbitts TH (1991) Novel genes for potential ligand-binding proteins in subregions of the olfactory mucosa. EMBO J. 10:2813-2819.

Distante C, Ancona N, Siciliano P (2003) Support vector machine for olfactory signals recognition. Sen. Actuat. B: Chem. 88:30-39.

Distel H, Hudson R. (1985) The contribution of the olfactory and tactile modalities to the nipplesearch behaviour of newborn rabbits. J. Comp. Physiol. 157: 599.

Dorries KM, Adkins RE, Halpern BP (1997) Sensitivity and behavioral responses to the pheromone androstenone are not mediated by the vomeronasal organ in domestic pigs. Brain Behav. Evol. 49:53-62.

Drafts B (2001) Acoustic wave technology sensors. IEEE Trans. 49:795-802.

Ellington AD, Szostak JW (1992) Selection in vitro of single-stranded DNA molecules that fold into specific ligand-binding structures. Nature. 355:850-852.

Feigon J, Dieckmann T, Smith FW (1996) Aptamer structures from A to $\zeta$. Chem. Biol. 3:611617.

Footner PK, Richards BP, and Yates RB (1987) Purple plague: Eliminated or just forgotten? Quality and Reliability Engineering International 3:177-184.

Fu J, Li G, Qin Y, Freeman WJ (2007) A pattern recognition method for electronic noses based on an olfactory neural network. Sens. Actua. B: Chem. 125:489-497. 
Gajurel P, Aldridge M, Glinka Y, Borisov P, Daly K, Lederman D (2016) The effect of gate -bias stress and light illumination on the performance of $\mathrm{ZnO}$ thin-film field effect transistors. APS March Meeting. X47.014.

Gao D, Liu F, Wang J (2012) Quantitative analysis of multiple kinds of volatile organic compounds using hierarchical models with an electronic nose. Sens. Actuat. B: Chemical. 161:578-586.

Gordan R, Gwathmey JK, Xie L-H (2015) Autonomic and endocrine control of cardiovascular function. World J. Cardiol. 7:204-214.

Grate JW (2000) Acoustic Wave Microsensor Arrays for Vapor Sensing. Chem. Rev. 100:262748.

Hagen JA, Kim SN, Bayraktaroglu B, Kelley-Loughnane N, Naik RR, Stone MO (2010) DNA aptamer functionalized zinc oxide field effect transistors for liquid state selective sensing of small molecules. Proc. Spie. 7759, Biosensing III, 775912.

Hagleitner C, Heirlemann A, Baltes H (2002) Single-chip CMOS capacitive gas sensor for detection of volatile organic compounds. IEEE Sensors Conference. 2:1428-1431.

Hallem EA, Carlson JR (2006) Coding of odors by a receptor repertoire. Cell 125:143-160.

Hildebrand JG, Shepard GM (1997) Mechanisms of olfactory discrimination: converging evidence for common principles across phyla. Ann. Rev. Neurosci. 20:595-631.

Hoon MA, Adler E, Lindemeier J, Battey JF, Ryba NJP, Zuker CS (1999) Putative mammalian taste receptors: a class of taste-specific GPCRs with distinct topographic selectivity. Cell. 96:541-551.

Horner G, Hierold C (1991) Gas analysis by partial model building. Sens. Actuat. B: Chem. 2:173-184.

Huang TC, Gilles R, Will G (1993) Thin-film thickness and density determination from x-ray reflectivity data using a conventional powder diffractometer. Thin Solid Films. 230:99-101.

Huizenga DE, Szostak JW (1995) A DNA aptamer that binds adenosine and ATP. Biochem. 34:656-665.

Ibarra AAG, Wrobel K, Escobosa ARC, Elguera JCT, Garay-Sevilla ME, Wrobel K (2015) Determination of putrescine, cadaverine, spermidine and spermine in different chemical matrices by high performance liquid chromatography-electrospray ionization-ion trap tandem mass spectrometry (HPLC-ESI-ITMS/MS). J. Chromatogr. B. 1002:176-184.

Joyce GF (1989) Amplification, mutation and selection of catalytic RNA. Gene. 82:83-87.

Jun J, Lee JS, Shin DH, Jang J (2014) Aptamer-functionalized hybrid carbon nanofiber FETtype electrode for a highly sensitive and selective platelet-derived growth factor biosensor. ACS Appl. Mater. Interfaces. 6:13859-13865.

Kahng D (1963) U. S. Patent No. 3,102,230. Washington, DC: U.S. Patent and Trademark Office. [in text: [U.S. Patent No. 3,102,230, 1963]

Kemp G (1998) Capillary Electrophoresis. Biotechnol. Appl. Biochem. 27:9-17.

Kepchia D, Sherman B, Haddad R, Luetje CW (2017) Mammalian odorant receptor tuning breadth persists across distinct odorant panels. PLoS ONE. 12:e0185329. 
King WH (1964) Piezoelectric sorption detector. Anal. Chem. 36:1735-1739.

Klug SJ, Famulok M (1994) All you wanted to know about SELEX. Mol. Biol. Rep. 20: 97-107.

Knight R, Yarus M (2003) Finding specific RNA motifs: Function in a zeptomole world? RNA. 9:218-230.

Larsson MC, Domingos AI, Jones WD, Chiappe ME, Amrein H, Vosshall LB (2004) Or83b encodes a broadly expressed odorant receptor essential for Drosophila olfaction. Neuron 43:703-714.

Larter NK, Sun JS, Carlson JR (2016) Organization and function of Drosophila odorant binding proteins. eLIFE 5:e20242.

Li M, Wang H, Sun L, Zhao G, Huang X (2016) Application of electronic nose for measuring total volatile basic nitrogen and total viable counts in packaged pork during refrigerated storage. J.Food Sci. 81:M906-M912.

Lin CH, Patel DJ (1997) Structural basis of DNA folding and recognition in an AMP-DNA aptamer complex: distinct architectures but common recognition motifs for DNA and RNA aptamers complexed to AMP. Chem. Biol. 4:817-832.

Lin MS, Tseng TF (1998) Chromium(III) hexacyanoferrate(II)-based chemical sensor for the cathodic determination of hydrogen peroxide. Analyst. 123:159-163.

Lucklum R, Henning B, Hauptmann P, Schierbaum KD, Vaihinger S, Göpel W (1991) Quartz microbalance sensors for gas detection. Sens. Actuat. A: Phys. 27:705-710.

Lundström I, Shivaraman MS, Svenson CS, Lundkvist L (1975) Hydrogen sensitive MOSFET. Appl. Phys. Lett. 26:55-57.

Luo M, Katz LC (2004) Encoding pheromonal signals in the mammalian vomeronasal system. Curr. Opin. Neurobiol. 14:428-310.

Menco BPM, Farbman Al (1992) Ultrastructural evidence for multiple mucous domains in frog olfactory epithelium. Cell Tissue Res. 270:47-56.

Meyer E (1992) Atomic force microscopy. Progress in Surface Science. 41:3-49.

Missbach C, Dweck HKM, Vogel H, Vilcinskas A, Stensmyr MC, Hansson BS, Grosse-Wilde E (2014) Evolution of insect olfactory receptors. eLife 3:e02115.

Mitrovics J, Ulmer H, Weimar U, Göpel W (1998) Modular sensor systems for gas sensing and odor monitoring: the MOSES concept. Acc. Chem. Res. 31:307-315

Mombaerts (2004) Genes and ligands for odorant, vomeronasal and taste receptors. Nat. Rev. Neurosci. 5:263-278.

Mortimer D, Fothergill T, Pujic Z, Richards LJ, Goodhill GJ (2008) Growth cone chemotaxis. Trends Neurosci. 31:90-98.

Mosing RK, Bowser MT (2009) Isolating aptamers using capillary electrophoresis-SELEX (CESELEX). Methods Mol. Biol. 535:33-43.

Moutin M-J, Cuillel M, Rapin C, Miras R, Anger M, Lompré A-M, Dupont Y (1994)

Measurements of ATP binding on the large cytoplasmic loop of the sarcoplasmic reticulum CA2+-ATPase overexpressed in Escherichia coli. J. Biol. Chem. 269:11147-11159. 
Nabity JC (2018) JC Nabity Lithography Systems. Retrieved from: https://www.jcnabity.com.

NIOSH (1994) NMAM 2500: Methyl Ethyl Ketone. https://www.cdc.gov/niosh/docs/2003154/pdfs/2500.pdf

NIOSH (1988) Occupational Safety and Health Guideline for 2-Butanone. NIOSH/OSHA Occupational Health Guidelines for Chemical Hazards- Vol. I, II and III. https://www.cdc.gov/niosh/docs/81-123/pdfs/0069.pdf

Novotny MV (2003) Pheromones, binding proteins and receptor responses in rodents. Biochem. Soc. Trans. 31:117-122.

Nylander C, Armgarth M, Lundström I (1983) An ammonia detector based on a conducting polymer. Anal. Chem. Symp. Ser. 17:203-207.

Orts J, Llobet E, Vilanova X, Brezmes J, Correig X (1999) Selective methane detection under varying moisture conditions using static and dynamic sensor signals. Sens. Actuat. B: Chem. 60:106-117.

Pan ZW, Dai ZR, Wang ZL (2001) Nanobelts of semiconducting oxides. Science 291:19471949.

Park S-K, Shanbhag SR, Wang Q, Hasan G, Steinbrecht RA, Pikielny CW (2000) Expression patterns of two putative odorant-binding proteins in the olfactory organs of Drosophila melanogaster have different implications for their functions. Cell Tissue Res. 300:181-192.

Pelosi P (1996) Perireceptor events in olfaction. J. Neurobiol. 30:3-19.

Persaud KC, Dodd G (1982) Analysis of discrimination mechanisms in the mammalian olfactory system using a model nose. Nature. 299:352-355.

Persaud KC, Dodd G (1982) Analysis of discrimination mechanisms in the mammalian olfactory system using a model nose. Nature. 299:352-355.

Plum TJ, Saxena V, Jessing RJ (2006) Design of a MEMS capacitive chemical sensor based on polymer swelling. IEEE Workshop on Microelectronics and Electron Devices.

Qian J, Lou X, Zang Y, Xiao Y, Soh HT (2009) Generation of highly specific aptamers via micromagnetic selection. Anal. Chem. 81:5490-5495.

Rebello MR, McTavish TS, Willhite DC, Short SM, Shepherd GM, Verhagen JV (2014) Perception of odors linked to precise timing in the olfactory system. PLoS Biol, 12:e1002021.

Ressler KJ, Sullivan SL, Buck LB (1994) Information coding in the olfactory system: Evidence for a stereotyped and highly organized epitope map in the olfactory bulb. Cell. 79:1245-1255.

Röck F, Barsan N, Weimar U (2008) Electronic nose: current status and future trends. Chem. Rev., 108:705-725.

Rytz R, Croset V, Benton R (2013) lonotropic receptors (IRs): chemosensory ionotropic glutamate receptors in Drosophila and beyond. Insect Biochem. Molec. 43:888-897.

Sassanfar M, Szostak JW (1993) An RNA motif that binds ATP. Nature. 364:550-553.

Silbering AF, Rytz R, Grosjean Y, Abuin L, Ramdya P, Jefferis GSXE, Benton R (2011) Complementary function and integrated wiring of the evolutionarily distinct Drosophila olfactory subsystems. J. Neurosci. 31:13357-13375. 
So H-M, Won K, Kim YH, Kim B-K, Ryu BH, Na PS, Kim H, Lee J-O (2005) JACS 172:1190611907.

Spors H, Wachowiak M, Cohen LB, Friedrich RW (2006) Temporal dynamics and latency patterns of receptor neuron input to the olfactory bulb. J Neurosci, 26:1247-1259.

Staii C, Johnson AT Jr, Chen M, Gelperin A (2005) DNA-decorated carbon nanotubes for chemical sensing. Nano Lett. 5:1774-1778.

Steinbrecht RA (1998) Odorant-binding proteins: expression and function. Ann. N.Y. Acad. Sci. 855:323-332.

Stensmyr MC, Dweck HKM, Farhan A, Ibba I, Strutz A, Mukunda L, Linz J, Grabe V, Steck K, Lavista-Llanos S, Wicher D, Sachse S, Knaden M, Becher PG, Seki Y, Hansson BS (2012) A conserved dedicated olfactory circuit for detecting harmful microbes in Drosophila. Cell 151:1345-1357.

Stoltenburg R, Reinemann C, Strehlitz B (2005) FluMag-SELEX as an advantageous method for DNA aptamer selection. Anal. Bioanal. Chem. 383:83-91.

Suryanarayana C, Norton MG (1998) X-ray diffraction: a practical approach. New York, NY: Plenum Press.

Taguchi N. (1972) U. S. Patent No. 3,695,848. Washington, DC: U.S. Patent and Trademark Office. [in text: [U.S. Patent No. 3,695,848, 1972]

Tuerk C, Gold L (1990) Systematic evolution of ligands by exponential enrichment: RNA ligands to bacteriophage T4 DNA polymerase. Science 249:505-510.

Vassar R, Chao K, Sitcheran R, Nuñez JM, Vosshall LB, Axel R (1994) Topographic organization of sensory projections to the olfactory bulb. Cell 79:981-991.

Vosshall LB, Amrein H, Morozov PS, Rzhetzky A, Axel R (1999) A spatial map of olfactory receptor expression in the Drosophila antenna. Cell 96:725-736.

Wang B, Li B, Deng Q, Dong S (1998) Amperometric glucose biosensor based on sol-gel organic-inorganic hybrid material. Anal. Chem. 70:3170-3174.

Wicher D, Schäfer R, Bauernfeind, Stensmyr MC, Heller R, Heinemann SH, Hansson BS (2008) Drosophila odorant receptors are both ligand-gated and cyclic-nucleotide-activated cation channels. Nature 452:1007-1012.

Wiedman GR, Zhao Y, Mustaev A, Ping J, Vishnubhotla R, Johnson ATC, Perlin DS (2017) An aptamer-based biosensor for the azole class of antifungal drugs. mSphere 2:e00274-17.

Wesson DW, Carey RM, Verhagen JV, Wachowiak M (2008) Rapid encoding and perception of novel odors in the rat. PLoS Biol. 6:e82.

Wilson AD, Baietto M (2009) Applications and advances in electronic-nose technologies. Sensors. 9:5099-5148.

Wilson RI, Turner GC, Laurent G (2004) Transformation of olfactory representations in the Drosophila antennal lobe. Science, 303:366-370.

Winquist F, Spetz A, Armgarth M, Lundström I, Danielsson B (1985) Biosensors based on ammonia sensitive metal-oxide-semiconductor structures. Sens. Actuat. 8:91-100. 
Wohltjen H, Dessy R (1979) Surface acoustic wave probe for chemical analysis. Anal. Chem. 51:1458-1475.

Wong AM, Wang JW, Axel R (2002) Spatial representation of the glomerular map in the Drosophila protocerebrum. Cell 109:229-241.

Yamada Y, Yamashita K, Masuoka Y, Seno Y (2001) Zn-Sn-Sb-O thin film sensor for ppmlevel NO2 detection. Sens. Actuat. B: Chem. 77:12-15.

Yamazoe N, Kurokuwa Y, Seiyama T (1983) Effects of additives on semiconductor gas sensors. Sens. Actuat. 4:283-289. 